\title{
Cascade assembly of 4,5,6,7-tetrahydroindole from cyclohexanone oxime and acetylene in KOH/DMSO superbase medium: a quantum chemical study
}

Alexander S. Bobkov, ${ }^{\mathrm{a}, \mathrm{b}}$ Nadezhda M. Vitkovskaya, ${ }^{\mathrm{a}^{*}}$ Boris A. Trofimov $^{\mathrm{b}}$

a Laboratory of Quantum Chemical Modeling of Molecular Systems, Irkutsk State University, 1 K. Marx St., 664003 Irkutsk, Russian Federation, e-mail: vita@cc.isu.ru

b A.E. Favorsky Irkutsk Institute of Chemistry, Siberian Branch of the Russian Academy of Sciences, 1 Favorsky St., 664033 Irkutsk, Russian Federation

\section{Table of Contents}

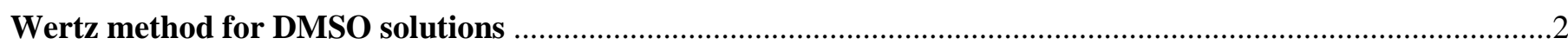

Potential Energy Surface of all stages of 4,5,6,7-tetrahydroindole formation and its vinylation .........................3

Description of high-barrier mechanisms of $\mathbf{3 H}$-pyrrole formation.............................................................

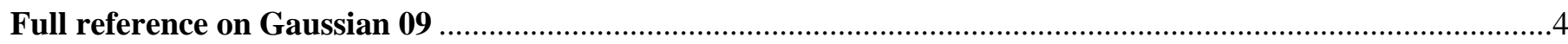

Total energies, enthalpies and Gibbs free energies (a.u.) calculated in the frame of B2PLYP/6$311+G^{* *} / / B 3 L Y P / 6-31+G^{*}$, Cartesian coordinates $(\AA)$ of stationary points 


\section{Wertz method for DMSO solutions}

Wertz ${ }^{1}$ approach suggests that all solutes lose the same fraction of entropy when dissolved in water. Abraham ${ }^{2}$ have shown that it takes place for other solvents. Wertz also argues that the same amount of entropy is lost when the ions are solvated. This allows one to introduce appropriate corrections for an arbitrary solvent.

Wertz method for DMSO solutions is composed of several steps. In the first stage a solute is treated as an ideal gas and compressed from 1atm to a hypothetical ideal gas state with the concentration equal to that of the liquid state $(\mathrm{d}=1.100 \mathrm{~g} / \mathrm{ml}, 298 \mathrm{~K}, 14.08 \mathrm{M})$. This entropy change can be estimated as

$$
\Delta S_{1}=-R \ln \frac{P_{2}}{P_{1}}=-R \ln (22.4 \cdot 14.08)=-47.85 \quad \mathrm{~J} \mathrm{~mol}^{-1} \mathrm{~K}^{-1} .
$$

The next step is the conversion of this hypothetical state to the final liquid state. The fraction of entropy lost in second step is defined as a coefficient, $\alpha$.

$$
\begin{aligned}
& \alpha=\frac{\Delta S_{2}}{S_{g}}=\frac{S_{g}-S_{l}^{0}}{S_{g}}=\frac{S_{g}^{0}+\Delta S_{1}-S_{l}^{0}}{S_{g}}, \\
& \Delta S_{2}=\alpha S_{g}=\alpha S_{g}^{0}+\alpha \Delta S_{1}
\end{aligned}
$$

The entropy of liquid DMSO $S_{l}^{0}=188.78 \mathrm{~J} \mathrm{~mol}^{-1} \mathrm{~K}^{-1}$ (standard state). ${ }^{3}$ The entropy of vaporization can be estimated from the normal heat of vaporization $(52.9 \mathrm{~kJ} / \mathrm{mol})^{4}$ and boiling temperature $(462 \mathrm{~K}$ at $1 \mathrm{~atm})$ as $\Delta S_{\text {vap }}^{0}=114.2 \mathrm{~J} \mathrm{~mol}^{-1} \mathrm{~K}^{-1}$, therefore, $S_{g}^{0}=303.0 \mathrm{~J} \mathrm{~mol}^{-1} \mathrm{~K}^{-1}$ and $\alpha$ $=0.26$.

The entropy change from the gas state of any given molecule $\mathbf{M}$ in standard state to its $1 \mathbf{M}$ state in DMSO is composed of three steps. The first is the compression of ideal $\mathbf{M}$ gas in standard state to a hypothetical ideal gas state with the concentration equal to that of the solvent liquid state $(14.08 \mathrm{M})$ that gives the same $\Delta S_{1}=-47.85 \mathrm{~J} \mathrm{~mol}^{-1} \mathrm{~K}^{-1}$. Conversion of the hypothetical ideal gas state to a hypothetical liquid state brings $\Delta S_{2}=\alpha S_{g}=\alpha S_{g}^{0}+\alpha \Delta S_{1}$. The fraction of entropy loss in this step is assumed to be equal to $\alpha$. Finally, expansion of the hypothetical liquid state to the $1 \mathrm{M}$ state in DMSO results in $\Delta S_{3}=R \ln (14.08)=21.99 \mathrm{~J} \mathrm{~mol}^{-1} \mathrm{~K}^{-1}$. The calculated gas phase entropy of $\mathbf{M}$ in standard state is then converted to the corresponding entropy in its $1 \mathrm{M}$ state in DMSO according to the following equation: $S_{l}^{0}=S_{g}^{0}+\Delta S_{1}-\Delta S_{2}+\Delta S_{3}=0.74 \times S_{g}^{0}-13.41 \mathrm{~J} \mathrm{~mol}^{-1} \mathrm{~K}^{-1}$,

For a bimolecular reaction it results in changing

$T \Delta S_{l}=0.74 \times T \Delta S_{g}-0.95 \mathrm{kcal} / \mathrm{mol}$.

\section{References:}

(1) Wertz, D. H. Relationship between the Gas-Phase Entropies of Molecules and Their Entropies of Solvation in Water and 1-Octanol. J. Am. Chem. Soc. 1980, 102 (16), 53165322. https://doi.org/10.1021/ja00536a033.

(2) Abraham, M. H. Relationship between Solution Entropies and Gas Phase Entropies of Nonelectrolytes. J. Am. Chem. Soc. 1981, 103 (22), 6742-6744. https://doi.org/10.1021/ja00412a036.

(3) Clever, H. L.; Westrum, E. F. Dimethyl Sulfoxide and Dimethyl Sulfone. Heat Capacities, Enthalpies of Fusion, and Thermodynamic Properties. J. Phys. Chem. 1970, 74 (6), 13091317. https://doi.org/10.1021/j100701a027.

(4) Douglas, T. B. Vapor Pressure of Methyl Sulfoxide from 20 to $50^{\circ}$. Calculation of the Heat of Vaporization. J. Am. Chem. Soc. 1948, 70 (6), 2001-2002.

https://doi.org/10.1021/ja01186a005. 


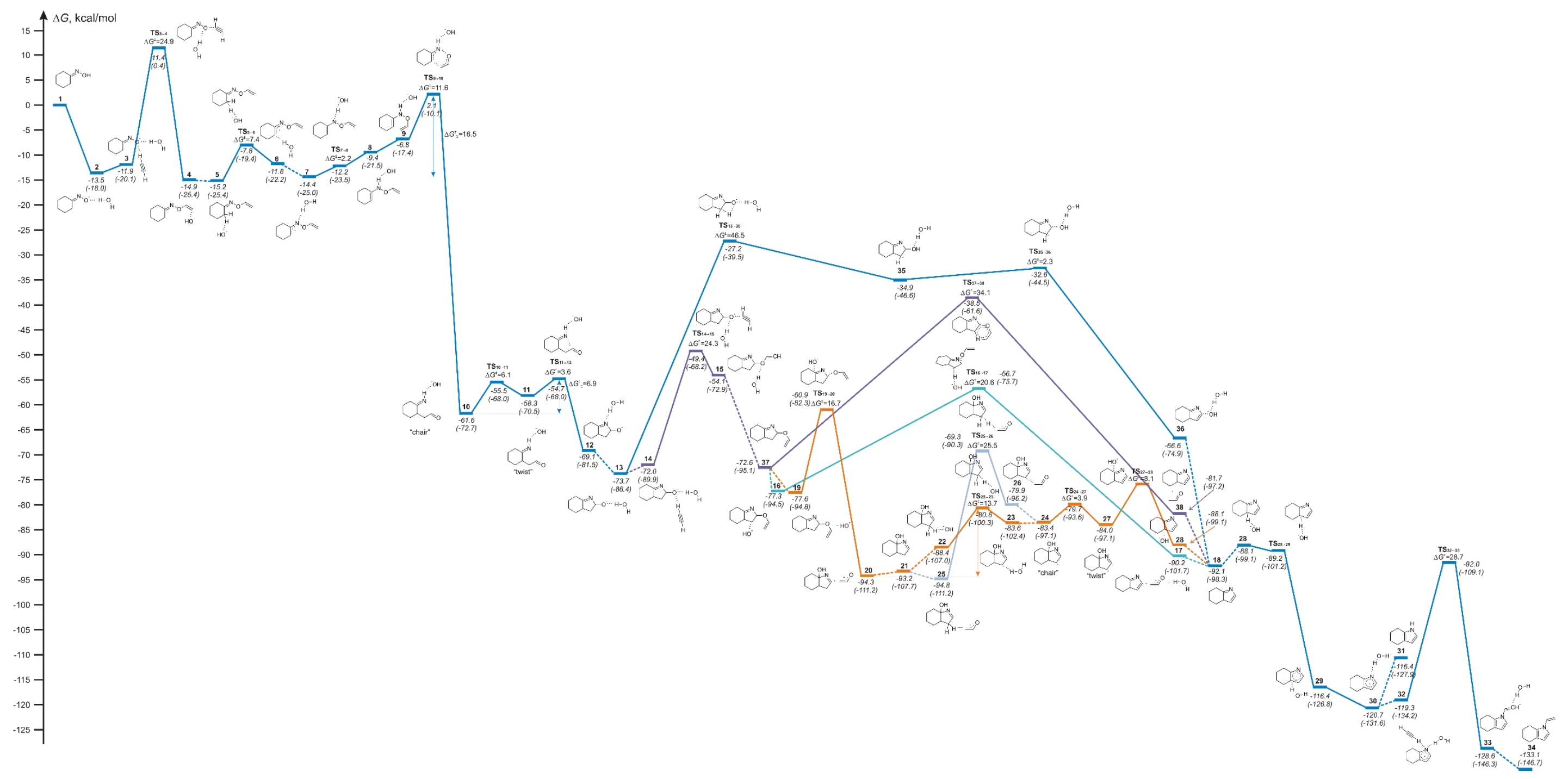




\section{Description of high-barrier mechanisms of 3H-pyrrole formation}

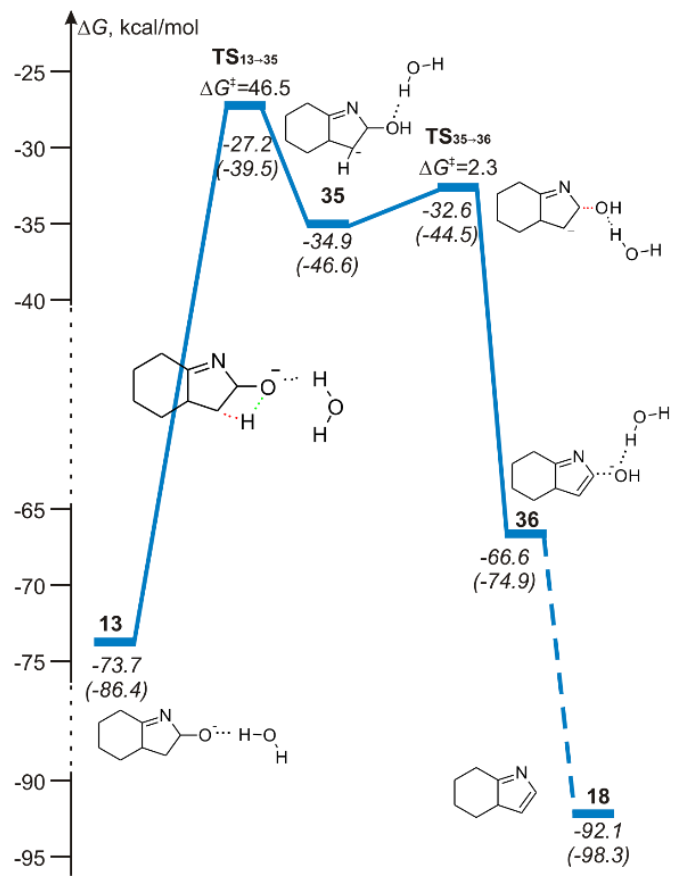

Mechanism 3. It essentially represent an intramolecular dehydration. Its most energetically demanding step is an intramolecular proton transfer from position 4 of the pyrroline ring to the alcoholate, resulting in the formation of a negatively charged carbon atom. The reaction is characterized by a notably high activation barrier $\Delta G^{*}=46.5 \mathrm{kcal} / \mathrm{mol}\left(\mathrm{TS}_{13 \rightarrow 35}\right)$. The resulting intermediate, $C$-centered anion $\mathbf{3 5}$ is unstable with respect to the initial $O$-centered anion by $39.8 \mathrm{kcal} / \mathrm{mol}$. This unstable intermediate via a small barrier $\Delta G^{*}=2.3$ $\mathrm{kcal} / \mathrm{mol}$ eliminates a hydroxide group, yielding $3 \mathrm{H}$ pyrrole. The thermal effect of the formation of $3 \mathrm{H}$ pyrrole 16 from 5-hydroxipyrroline amounts to $\Delta H=-$ $11.9 \mathrm{kcal} / \mathrm{mol}$. However, the activation barrier of 46.5 $\mathrm{kcal} / \mathrm{mol}$ cannot be overcome under the experimental conditions ( $1 \mathrm{~atm}, \sim 60-80^{\circ} \mathrm{C}$ ).

Mechanism 4. Neutral 5-vinyloxypyrroline 37 eliminates acetaldehyde via $\mathrm{TS}_{37 \rightarrow 38}$. A simultaneous rupture of the chemical bonds $\mathrm{C}-$ $\mathrm{O}$ and $\mathrm{C}$ (cycle)-H and the acetaldehyde molecule formation are occurred. It proceeds with an activation barrier of $\Delta G^{*}=34.1$ $\mathrm{kcal} / \mathrm{mol}$. However, overcoming such an activation barrier also seems to be impossible under the experimental conditions.

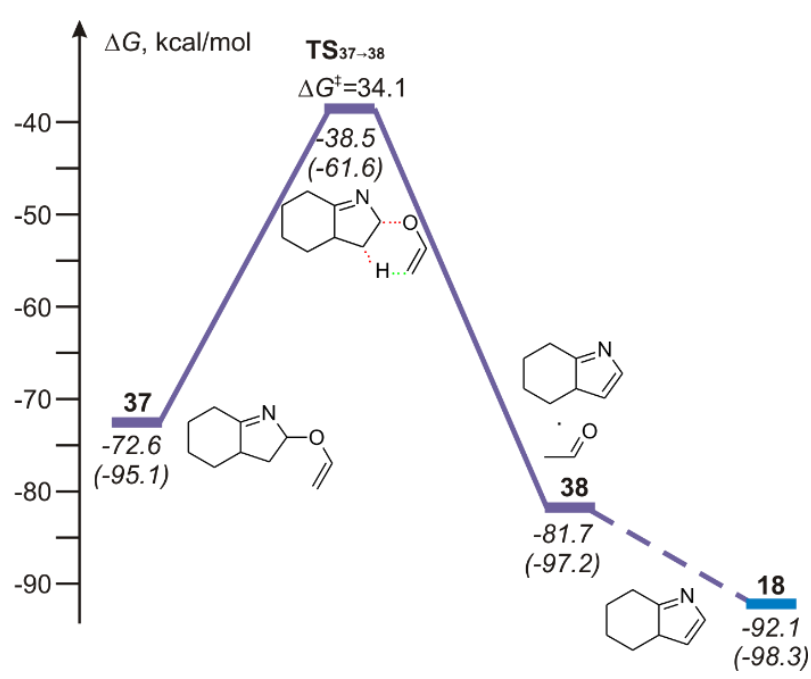

\section{Full reference on Gaussian 09}

M.J. Frisch, G.W. Trucks, H.B. Schlegel, G.E. Scuseria, M.A. Robb, J.R. Cheeseman, G. Scalmani, V. Barone, B. Mennucci, G.A. Petersson, H. Nakatsuji, M. Caricato, X. Li, H.P. Hratchian, A.F. Izmaylov, J. Bloino, G. Zheng, J.L. Sonnenberg, M. Hada, M. Ehara, K. Toyota, R. Fukuda, J. Hasegawa, M. Ishida, T. Nakajima, Y. Honda, O. Kitao, H. Nakai, T. Vreven, J.A. Montgomery, Jr., J.E. Peralta, F. Ogliaro, M. Bearpark, J.J. Heyd, E. Brothers, K. N. Kudin, V.N. Staroverov, T. Keith, R. Kobayashi, J. Normand, K. Raghavachari, A. Rendell, J.C. Burant, S.S. Iyengar, J. Tomasi, M. Cossi, N. Rega, J.M. Millam, M. Klene, J.E. Knox, J.B. Cross, V. Bakken, C. Adamo, J. Jaramillo, R. Gomperts, R.E. Stratmann, O. Yazyev, A.J. Austin, R. Cammi, C. Pomelli, J.W. Ochterski, R.L. Martin, K. Morokuma, V.G. Zakrzewski, G.A. Voth, P. Salvador, J.J. Dannenberg, S. Dapprich, A.D. Daniels, Ö. Farkas, J.B. Foresman, J.V. Ortiz, J. Cioslowski, and D.J. Fox, Gaussian 09, Revision C.01, Inc., Wallingford CT, 2010 
Total energies, enthalpies and Gibbs free energies (a.u.) calculated in the frame of B2PLYP/6$311+G * * / / B 3 L Y P / 6-31+G *$, Cartesian coordinates $(\AA)$ of stationary points

Cartesian coordinates

\begin{tabular}{llll}
\hline $\mathrm{O}$ & 0.0000000 & 0.0000000 & 0.1185800 \\
$\mathrm{H}$ & 0.0000000 & 0.7684560 & -0.4743190 \\
$\mathrm{H}$ & 0.0000000 & -0.7684560 & -0.4743190
\end{tabular}

\section{Water}

$\begin{array}{ll}E \text { (B2PLYP }) & -76.3979996 \\ E(\mathrm{~B} 2 P L Y P)+Z P E & -76.3769816 \\ E(\mathrm{~B} 2 P L Y P)+H_{\text {corr }} & -76.3732026 \\ E(\mathrm{~B} 2 \mathrm{PLYP})+G_{\text {corr }} & -76.3946506\end{array}$

\begin{tabular}{llll}
\hline $\mathrm{O}$ & 0.0000000 & 0.0000000 & 0.1079790 \\
$\mathrm{H}$ & 0.0000000 & 0.0000000 & -0.8638350
\end{tabular}

\begin{tabular}{llll}
\hline $\mathrm{C}$ & 0.0000000 & 0.0000000 & 0.6046290 \\
$\mathrm{C}$ & 0.0000000 & 0.0000000 & -0.6046290 \\
$\mathrm{H}$ & 0.0000000 & 0.0000000 & -1.6738930 \\
$\mathrm{H}$ & 0.0000000 & 0.0000000 & 1.6738930
\end{tabular}

Hydroxide ion

$\begin{array}{lc}E \text { (B2PLYP }) & \text { a.u. } \\ E \text { (B2PLYP })+Z P E & -75.766347 \\ E(\mathrm{~B} 2 \mathrm{PLYP})+H_{\text {corr }} & -75.754566 \\ E(\mathrm{~B} 2 \mathrm{PLYP})+G_{\text {corr }} & -75.774132\end{array}$




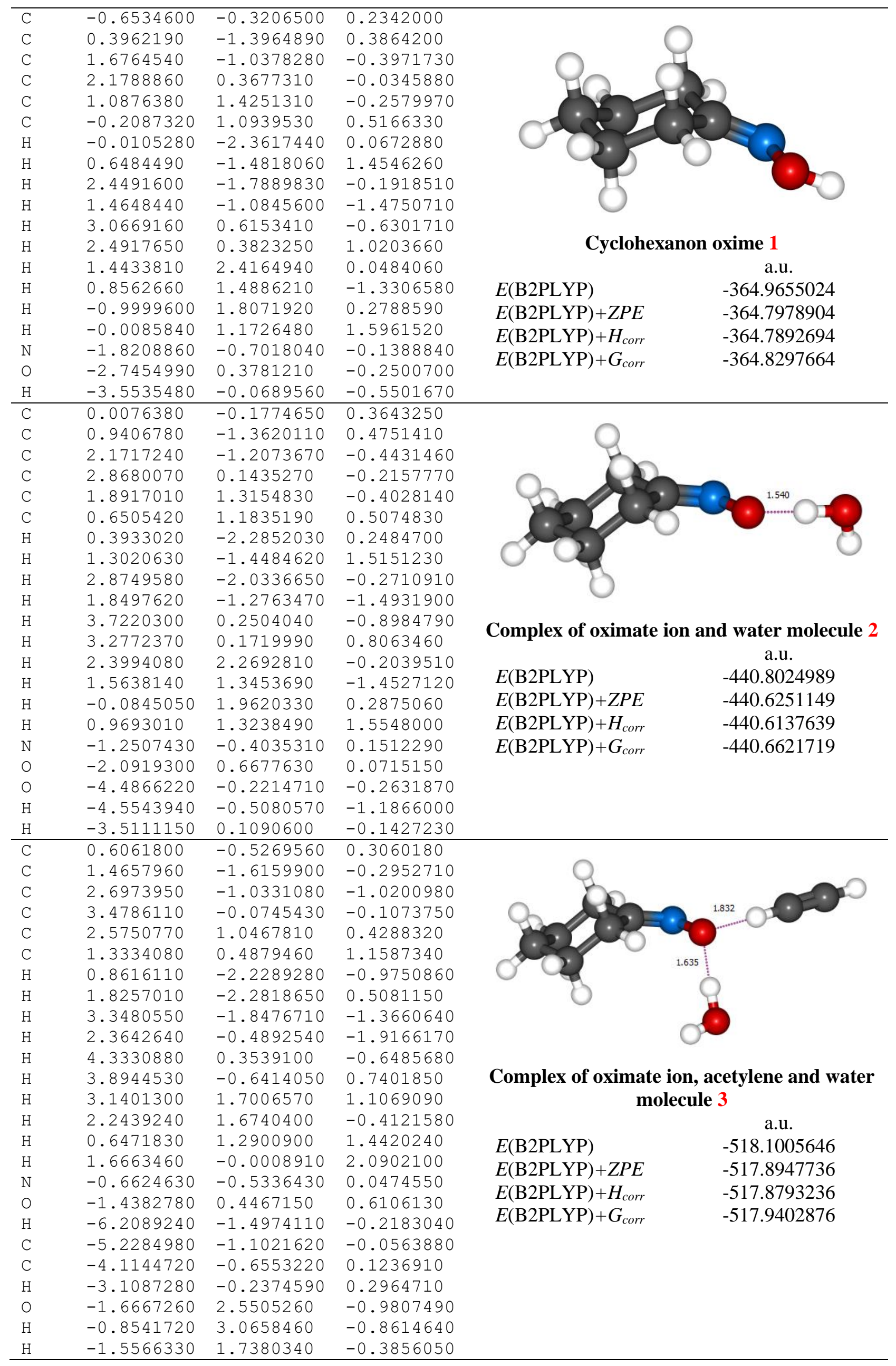




\begin{tabular}{llll}
\hline & & & \\
$\mathrm{C}$ & 0.5311720 & -0.6604890 & 0.2539760 \\
$\mathrm{C}$ & 1.6391480 & -1.5382140 & -0.2834560 \\
$\mathrm{C}$ & 2.7617050 & -0.6961740 & -0.9271090 \\
$\mathrm{C}$ & 3.2613640 & 0.3940470 & 0.0333570 \\
$\mathrm{C}$ & 2.1084920 & 1.2889190 & 0.5144640 \\
$\mathrm{C}$ & 0.9728120 & 0.4648450 & 1.1619660 \\
$\mathrm{H}$ & 1.2259920 & -2.2599180 & -0.9974520 \\
$\mathrm{H}$ & 2.0757320 & -2.1150460 & 0.5489680 \\
$\mathrm{H}$ & 3.5902300 & -1.3506860 & -1.2283830 \\
$\mathrm{H}$ & 2.3781940 & -0.2252770 & -1.8443310 \\
$\mathrm{H}$ & 4.0328910 & 1.0040140 & -0.4551070 \\
$\mathrm{H}$ & 3.7394170 & -0.0810960 & 0.9039180 \\
$\mathrm{H}$ & 2.4768030 & 2.0341910 & 1.2317360 \\
$\mathrm{H}$ & 1.7036560 & 1.8470300 & -0.3423890 \\
$\mathrm{H}$ & 0.1229510 & 1.0998980 & 1.4234930 \\
$\mathrm{H}$ & 1.3514240 & 0.0257560 & 2.0997510 \\
$\mathrm{~N}$ & -0.6800800 & -0.9268960 & -0.1037610 \\
$\mathrm{O}$ & -1.6737460 & -0.1207700 & 0.3970750 \\
$\mathrm{H}$ & -4.7753180 & 0.3826000 & 0.8331960 \\
$\mathrm{C}$ & -4.4763080 & -0.4738420 & 0.2428860 \\
$\mathrm{C}$ & -3.3463280 & -0.9283250 & -0.1028630 \\
$\mathrm{H}$ & -2.8340800 & -1.7091090 & -0.6431140 \\
$\mathrm{O}$ & -1.8442430 & 2.1990980 & -1.0185640 \\
$\mathrm{H}$ & -1.1203310 & 2.7556350 & -0.6926350 \\
$\mathrm{H}$ & -1.7754290 & 1.3490510 & -0.4987320 \\
& & &
\end{tabular}

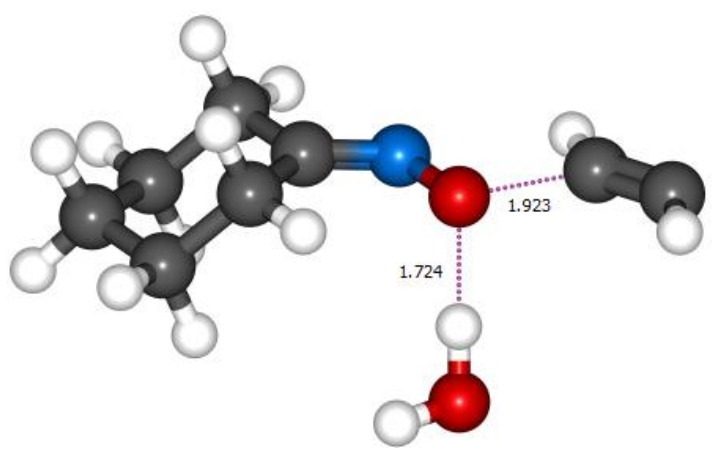

Transition state of oxime vinylation $\left(\mathrm{TS}_{3 \rightarrow 4}\right)$ $1310 \mathrm{~cm}^{-1}$

$E$ (B2PLYP)

$E(\mathrm{~B} 2 \mathrm{PLYP})+Z P E$

$E(\mathrm{~B} 2 \mathrm{PLYP})+H_{\text {corr }}$

$E(\mathrm{~B} 2 \mathrm{PLYP})+G_{\text {corr }}$

a.u.

$-518.06677$

$-517.860116$

$-517.845879$

$-517.900896$

\begin{tabular}{llll}
\hline & & & \\
$\mathrm{C}$ & -1.0414280 & -0.3238780 & -0.4114740 \\
$\mathrm{C}$ & -2.0854420 & -1.4158630 & -0.4225240 \\
$\mathrm{C}$ & -3.2124160 & -1.1137340 & 0.5877510 \\
$\mathrm{C}$ & -3.7917420 & 0.2929160 & 0.3766010 \\
$\mathrm{C}$ & -2.6935070 & 1.3642250 & 0.4461930 \\
$\mathrm{C}$ & -1.5501560 & 1.0892030 & -0.5576550 \\
$\mathrm{H}$ & -1.6156030 & -2.3838120 & -0.2203370 \\
$\mathrm{H}$ & -2.5186720 & -1.4639610 & -1.4332410 \\
$\mathrm{H}$ & -3.9970940 & -1.8738830 & 0.4899680 \\
$\mathrm{H}$ & -2.8130670 & -1.1967170 & 1.6085660 \\
$\mathrm{H}$ & -4.5632850 & 0.4986790 & 1.1293870 \\
$\mathrm{H}$ & -4.2864190 & 0.3401410 & -0.6051130 \\
$\mathrm{H}$ & -3.1109580 & 2.3582850 & 0.2451080 \\
$\mathrm{H}$ & -2.2766480 & 1.3946060 & 1.4627060 \\
$\mathrm{H}$ & -0.7417510 & 1.8120910 & -0.4382920 \\
$\mathrm{H}$ & -1.9412220 & 1.1966160 & -1.5807810 \\
$\mathrm{~N}$ & 0.1759670 & -0.7035330 & -0.2582760 \\
$\mathrm{O}$ & 1.0914860 & 0.3906600 & -0.2636640 \\
$\mathrm{H}$ & 3.2563360 & 1.8781780 & -0.0850920 \\
$\mathrm{C}$ & 3.4075220 & 0.8044990 & 0.0018090 \\
$\mathrm{C}$ & 2.3809240 & -0.0471990 & -0.0702680 \\
$\mathrm{H}$ & 2.4700270 & -1.1269770 & 0.0111840 \\
$\mathrm{O}$ & 6.4869350 & -0.3983800 & 0.3760020 \\
$\mathrm{H}$ & 7.3789530 & -0.7726550 & 0.4770430 \\
$\mathrm{H}$ & 4.4177460 & 0.4248970 & 0.1455230
\end{tabular}

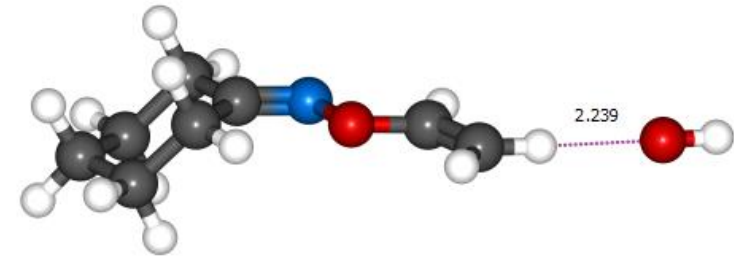

Complex of $\boldsymbol{O}$-vinyloxime and hydroxide ion 4

$E(\mathrm{~B} 2 \mathrm{PLYP})$

a.u.

$E$ (B2PLYP) $+Z P E$

$E$ (B2PLYP $)+H_{\text {corr }}$

$E(\mathrm{~B} 2 \mathrm{PLYP})+G_{c o r r}$
$-518.0763184$

$-517.8664554$

$-517.8519994$
$-517.9081554$ 


\begin{tabular}{llll}
\hline & & & \\
$\mathrm{C}$ & -0.0789920 & -0.3110920 & -0.4015550 \\
$\mathrm{C}$ & -1.1371080 & -0.9145720 & -1.2964850 \\
$\mathrm{C}$ & -2.1870960 & -1.6877230 & -0.4725130 \\
$\mathrm{C}$ & -2.7624520 & -0.8161130 & 0.6530780 \\
$\mathrm{C}$ & -1.6484190 & -0.2737790 & 1.5603320 \\
$\mathrm{C}$ & -0.5731740 & 0.5044330 & 0.7650380 \\
$\mathrm{H}$ & -0.6685740 & -1.5553390 & -2.0506670 \\
$\mathrm{H}$ & -1.6391870 & -0.0914650 & -1.8265960 \\
$\mathrm{H}$ & -2.9851720 & -2.0347970 & -1.1404150 \\
$\mathrm{H}$ & -1.7200440 & -2.5847750 & -0.0409320 \\
$\mathrm{H}$ & -3.4813420 & -1.3948750 & 1.2474060 \\
$\mathrm{H}$ & -3.3176370 & 0.0258190 & 0.2138920 \\
$\mathrm{H}$ & -2.0675750 & 0.3891860 & 2.3272430 \\
$\mathrm{H}$ & -1.1727600 & -1.1107440 & 2.0918290 \\
$\mathrm{H}$ & 0.2545550 & 0.7931270 & 1.4159420 \\
$\mathrm{H}$ & -1.0174340 & 1.4273840 & 0.3568470 \\
$\mathrm{~N}$ & 1.1372410 & -0.5621400 & -0.7309200 \\
$\mathrm{O}$ & 2.0781470 & 0.0549080 & 0.1579570 \\
$\mathrm{H}$ & 4.3058610 & 0.8391040 & 1.3523920 \\
$\mathrm{C}$ & 4.4141980 & 0.2099720 & 0.4735450 \\
$\mathrm{C}$ & 3.3605580 & -0.2466510 & -0.2109530 \\
$\mathrm{H}$ & 3.4318720 & -0.8764950 & -1.0935200 \\
$\mathrm{H}$ & 5.4143820 & -0.0503440 & 0.1455340 \\
$\mathrm{O}$ & -1.7630850 & 3.3461740 & -0.5782010 \\
$\mathrm{H}$ & -2.1432240 & 4.1636830 & -0.9434830 \\
& & &
\end{tabular}

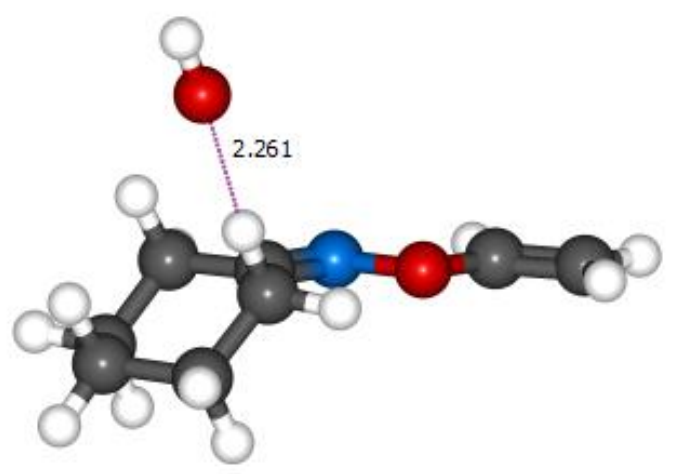

Complex of $O$-vinyloxime and hydroxide ion 5

$E$ (B2PLYP)

$E$ (B2PLYP) $+Z P E$

$E$ (B2PLYP) $+H_{\text {corr }}$

$E(\mathrm{~B} 2 \mathrm{PLYP})+G_{\text {corr }}$ a.u.

$-518.0888068$

$-517.8790968$

$-517.8645298$

$-517.9213458$
$-0.1643650$

$-1.2297410$

$-2.4947590$

$-3.0088570$

$-1.9345140$

$-0.5769170$

$-0.8085620$

$-1.5020270$

$-3.2630280$

$-2.2685850$

$-3.9239860$

$-3.2801920$

$-2.2820800$

$-1.8381990$

0.2097120

$-0.6926450$

1.0472060

2.0368910

4. 3225690

4.3821950

3. 2901740

3.3330520

5.3617250

$-0.8267050$

0.0410190
$-0.4709780$

$-1.2753070$

$-1.4778950$

$-0.1342500$

0.5562640

0.6665710

$-2.2340670$

$-0.7240520$

$-1.9814390$

$-2.1432400$

$-0.2822430$

0.5086550

1. 5597440

$-0.0068000$

1.0281850

1.7422830

$-0.8760340$

$-0.0419790$

0.9675230

0.0871860

$-0.4998860$

$-1.3815670$

$-0.3234450$

2. 7322160

3.1705690
$-0.2095570$

$-0.9452610$

$-0.0973140$

0.4346840

1. 2874440

0.5744050

$-1.2686510$

$-1.8582150$

$-0.6990440$

0.7498630

1.0246110

$-0.4163490$

1. 5737280

2. 2336270

1. 2427910

$-0.3426970$

$-0.5039500$

0.1851900

1. 1019200

0.4676010

$-0.0459360$

$-0.6825350$

0.2481090

$-1.0753560$

$-1.0545790$

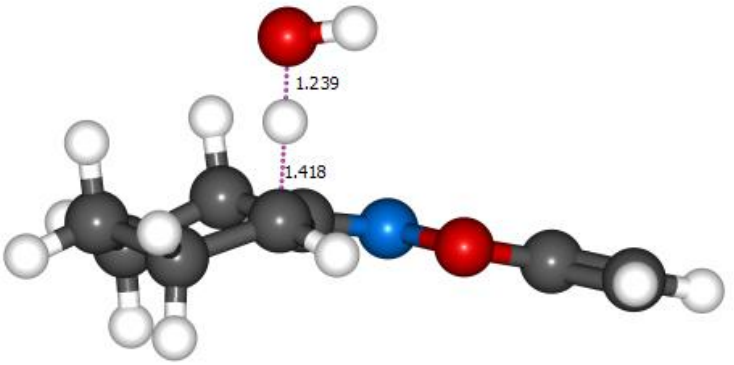

Transition state of the proton detachment

$\left(\mathrm{TS}_{5 \rightarrow 6}\right)$

$I 1338 \mathrm{~cm}^{-1}$

$E$ (B2PLYP)

a.u.

$E(\mathrm{~B} 2 \mathrm{PLYP})+Z P E$

$-518.1015163$

$E(\mathrm{~B} 2 \mathrm{PLYP})+H_{\text {corr }}$

$-517.8971343$

$-517.8835033$

$E(\mathrm{~B} 2 \mathrm{PLYP})+G_{\text {corr }}$ 


$\begin{array}{llll}\mathrm{C} & -0.2160240 & -0.5171670 & -0.1328000 \\ \mathrm{C} & -1.2912960 & -1.2524020 & -0.9351640 \\ \mathrm{C} & -2.6269040 & -1.3483240 & -0.1854350 \\ \mathrm{C} & -3.0607340 & 0.0333840 & 0.3179480 \\ \mathrm{C} & -2.0186260 & 0.5984040 & 1.2951470 \\ \mathrm{C} & -0.5924550 & 0.4628390 & 0.7838740 \\ \mathrm{H} & -0.9229900 & -2.2508280 & -1.2022690 \\ \mathrm{H} & -1.4526720 & -0.7186950 & -1.8841260 \\ \mathrm{H} & -3.3910010 & -1.7829720 & -0.8444070 \\ \mathrm{H} & -2.5222540 & -2.0304110 & 0.6723280 \\ \mathrm{H} & -4.0459640 & -0.0218980 & 0.8016390 \\ \mathrm{H} & -3.1658430 & 0.7091460 & -0.5447350 \\ \mathrm{H} & -2.2565010 & 1.6531990 & 1.5071870 \\ \mathrm{H} & -2.1467280 & 0.0771430 & 2.2646540 \\ \mathrm{H} & 0.1850060 & 0.8964190 & 1.4108740 \\ \mathrm{H} & -0.4731140 & 1.9622270 & -0.4991200 \\ \mathrm{~N} & 1.0066580 & -0.9122590 & -0.5229670 \\ \mathrm{O} & 2.0159720 & -0.1236480 & 0.2375600 \\ \mathrm{H} & 4.3277850 & 0.8668780 & 1.1455190 \\ \mathrm{C} & 4.3695850 & 0.0177770 & 0.4680910 \\ \mathrm{C} & 3.2604410 & -0.5478030 & -0.0432670 \\ \mathrm{H} & 3.3001920 & -1.3973410 & -0.7246230 \\ \mathrm{H} & 5.3430680 & -0.3819150 & 0.2047730 \\ \mathrm{O} & -0.4261930 & 2.7817430 & -1.0802740 \\ \mathrm{H} & 0.5122460 & 2.8598430 & -1.3155860 \\ & & & \end{array}$

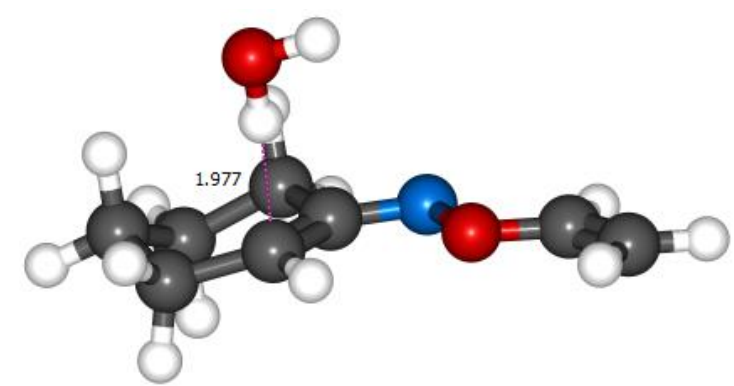

Complex of $O$-vinyloxime anion and water molecule 6

E(B2PLYP)

a.u.

$E(\mathrm{~B} 2 \mathrm{PLYP})+Z P E$

$-518.1163097$

$-517.9081337$

$-517.8936757$

$E$ (B2PLYP $)+H_{\text {corr }}$

$-517.9498527$

\begin{tabular}{|c|c|c|c|}
\hline C & -0.4451950 & 0.0306690 & -0.1053130 \\
\hline C & -1.4292400 & 1.1135180 & -0.5451790 \\
\hline C & -2.8374660 & 0.5647690 & -0.8102980 \\
\hline C & -3.3002470 & -0.3102310 & 0.3596020 \\
\hline C & -2.3677700 & -1.5203860 & 0.5261280 \\
\hline $\mathrm{C}$ & -0.9011260 & -1.1535590 & 0.4136990 \\
\hline $\mathrm{H}$ & -1.0383750 & 1.6156490 & -1.44 \\
\hline $\mathrm{H}$ & -1.4842510 & 1.8867100 & 0.2365680 \\
\hline $\mathrm{H}$ & -3.5345020 & 1.3977250 & -0.97 \\
\hline $\mathrm{H}$ & -2.8355680 & -0.0344650 & -1.733528 \\
\hline $\mathrm{H}$ & -4.3364570 & -0.6441130 & 0.2104940 \\
\hline $\mathrm{H}$ & -3.2889620 & 0.2940550 & 1.279 \\
\hline $\mathrm{H}$ & -2.5715380 & -2.0041780 & 1.4950440 \\
\hline $\mathrm{H}$ & -2.6450780 & -2.2780810 & -0.232107 \\
\hline $\mathrm{H}$ & -0.1777190 & -1.9078530 & 0.7149310 \\
\hline $\mathrm{N}$ & 0.8411670 & 0.4595830 & -0.302192 \\
\hline O & 1.7681720 & -0.5487130 & 0.2723390 \\
\hline $\mathrm{H}$ & 4.0776410 & -1.7466980 & 0.8840000 \\
\hline $\mathrm{C}$ & 3.9359140 & -1.4132690 & -0.140827 \\
\hline $\mathrm{C}$ & 2.8136450 & -0.7858600 & -0.541441 \\
\hline $\mathrm{H}$ & 2.6733090 & -0.4339250 & -1.56352 \\
\hline $\mathrm{H}$ & 4.7214400 & -1.6161280 & -0.861129 \\
\hline $\mathrm{H}$ & 1.3656380 & 1.9514980 & 0.3765670 \\
\hline O & 1.7103160 & 2.8176480 & 0.7847270 \\
\hline $\mathrm{H}$ & 2.5472590 & 2.9973280 & 0.3286880 \\
\hline
\end{tabular}

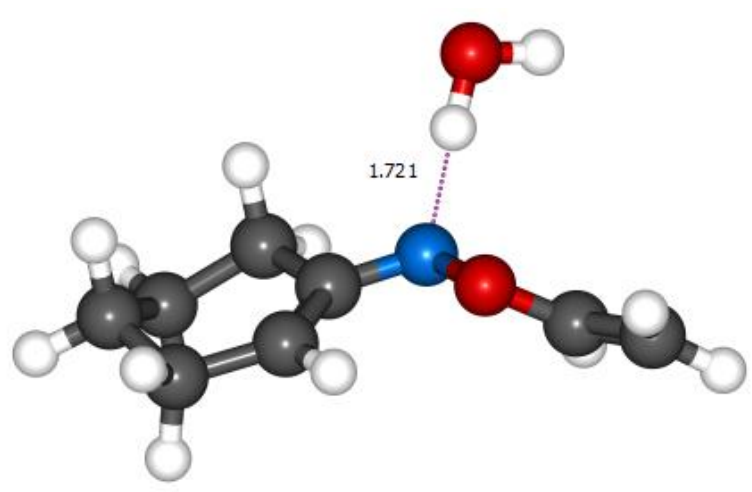

Complex of $\boldsymbol{O}$-vinyloxime anion and water molecule 7

$E$ (B2PLYP)

$E(\mathrm{~B} 2 \mathrm{PLYP})+Z P E$

$E(\mathrm{~B} 2 \mathrm{PLYP})+H_{\text {corr }}$

$E(\mathrm{~B} 2 \mathrm{PLYP})+G_{\text {corr }}$ a.u.

$-518.1189261$

$-517.9104581$

$-517.8962041$

$-517.9521291$ 


\begin{tabular}{llll}
\hline & & & \\
$\mathrm{C}$ & -0.3918640 & 0.0989180 & -0.0920830 \\
$\mathrm{C}$ & -1.4564110 & 1.0832370 & -0.5559840 \\
$\mathrm{C}$ & -2.8136170 & 0.4089190 & -0.8011800 \\
$\mathrm{C}$ & -3.1823870 & -0.5089590 & 0.3699960 \\
$\mathrm{C}$ & -2.1395000 & -1.6265210 & 0.5269800 \\
$\mathrm{C}$ & -0.7172820 & -1.1211530 & 0.4051770 \\
$\mathrm{H}$ & -1.1106770 & 1.5862610 & -1.4688720 \\
$\mathrm{H}$ & -1.5661720 & 1.8748270 & 0.2002380 \\
$\mathrm{H}$ & -3.5841220 & 1.1758540 & -0.9547260 \\
$\mathrm{H}$ & -2.7679930 & -0.1850770 & -1.7261510 \\
$\mathrm{H}$ & -4.1823790 & -0.9395340 & 0.2261190 \\
$\mathrm{H}$ & -3.2224480 & 0.0890430 & 1.2929230 \\
$\mathrm{H}$ & -2.2798720 & -2.1268410 & 1.4972260 \\
$\mathrm{H}$ & -2.3357260 & -2.4069600 & -0.2302870 \\
$\mathrm{H}$ & 0.0780170 & -1.7946500 & 0.7153780 \\
$\mathrm{~N}$ & 0.8854530 & 0.6228960 & -0.2820980 \\
$\mathrm{O}$ & 1.8819030 & -0.2259090 & 0.3637440 \\
$\mathrm{H}$ & 4.0906290 & -1.6221660 & 0.8791280 \\
$\mathrm{C}$ & 3.8123890 & -1.4937430 & -0.1634960 \\
$\mathrm{C}$ & 2.7230080 & -0.8011950 & -0.5324190 \\
$\mathrm{H}$ & 2.4327110 & -0.6526820 & -1.5707630 \\
$\mathrm{H}$ & 4.4370430 & -1.9503680 & -0.9235730 \\
$\mathrm{H}$ & 1.0883300 & 1.7475560 & 0.1895870 \\
$\mathrm{O}$ & 1.3082260 & 2.9049870 & 0.6975640 \\
$\mathrm{H}$ & 2.1974360 & 3.1748180 & 0.4160460
\end{tabular}

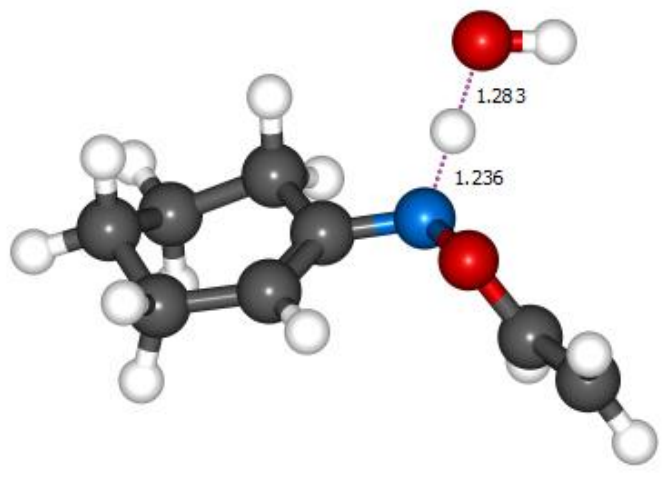

Transition state of protonation $\left(\mathrm{TS}_{7 \rightarrow 8}\right)$ $I 1037 \mathrm{~cm}^{-1}$

a.u.

$E$ (B2PLYP)

$E(\mathrm{~B} 2 \mathrm{PLYP})+Z P E$

$E(\mathrm{~B} 2 \mathrm{PLYP})+H_{\text {corr }}$

$E(\mathrm{~B} 2 \mathrm{PLYP})+G_{\text {corr }}$
$-518.1041673$

$-517.8994093$

$-517.8857273$

$-517.9399803$

\begin{tabular}{llll}
\hline & & & \\
$\mathrm{C}$ & 0.4033360 & 0.0728070 & 0.1149730 \\
$\mathrm{C}$ & 1.4625160 & 1.0832500 & 0.5170500 \\
$\mathrm{C}$ & 2.8341600 & 0.4285850 & 0.7370700 \\
$\mathrm{C}$ & 3.1668050 & -0.5389800 & -0.4053460 \\
$\mathrm{C}$ & 2.1261390 & -1.6670540 & -0.4807280 \\
$\mathrm{C}$ & 0.7074450 & -1.1617770 & -0.3355790 \\
$\mathrm{H}$ & 1.1400080 & 1.6057790 & 1.4275270 \\
$\mathrm{H}$ & 1.5321390 & 1.8553040 & -0.2632840 \\
$\mathrm{H}$ & 3.6028830 & 1.2068090 & 0.8257760 \\
$\mathrm{H}$ & 2.8286890 & -0.1228050 & 1.6887610 \\
$\mathrm{H}$ & 4.1724200 & -0.9597280 & -0.2756400 \\
$\mathrm{H}$ & 3.1738320 & 0.0172550 & -1.3544710 \\
$\mathrm{H}$ & 2.2331450 & -2.2075800 & -1.4324930 \\
$\mathrm{H}$ & 2.3416160 & -2.4134910 & 0.3033380 \\
$\mathrm{H}$ & -0.0963850 & -1.8448430 & -0.5985360 \\
$\mathrm{~N}$ & -0.8953280 & 0.5756100 & 0.3301260 \\
$\mathrm{O}$ & -1.8879440 & -0.2186630 & -0.3548070 \\
$\mathrm{H}$ & -4.0511010 & -1.6294550 & -0.9631900 \\
$\mathrm{C}$ & -3.8215880 & -1.5068570 & 0.0917080 \\
$\mathrm{C}$ & -2.7593610 & -0.8088500 & 0.5140790 \\
$\mathrm{H}$ & -2.5074160 & -0.6614710 & 1.5614170 \\
$\mathrm{H}$ & -4.4772180 & -1.9715680 & 0.8198360 \\
$\mathrm{H}$ & -1.0402910 & 1.5749440 & -0.0510620 \\
$\mathrm{O}$ & -1.2502630 & 3.0732170 & -0.6218240 \\
$\mathrm{H}$ & -2.1960810 & 3.2784050 & -0.7051870
\end{tabular}

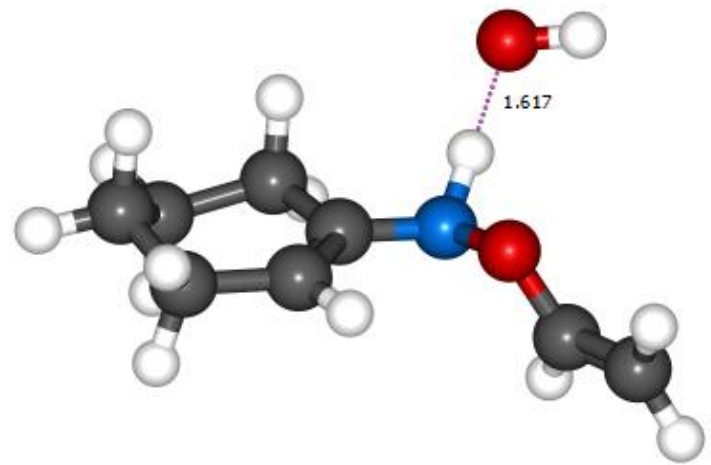

Complex of $O$-vinylhydroxylamine and hydroxide ion 8

$E$ (B2PLYP)

$E(\mathrm{~B} 2 \mathrm{PLYP})+Z P E$

$E(\mathrm{~B} 2 \mathrm{PLYP})+H_{\text {corr }}$

$E(\mathrm{~B} 2 \mathrm{PLYP})+G_{\text {corr }}$ a.u. $-518.0953447$ $-517.8865357$ $-517.8733027$ $-517.9259867$ 
$-0.3409970$

$-0.7342170$

$-2.2604820$

$-2.9526810$

$-2.6590920$

$-1.2054450$

$-0.2729270$

$-0.3132440$

$-2.5097600$

$-2.6352160$

$-4.0359420$

$-2.5869960$

$-2.9471470$

$-3.2882730$

$-0.8470380$

0.9762850

1. 9411980

3. 5772540

3.3929950

2. 5755470

2.3710000

3. 9015490

1.0828830

1. 1821400

1.7893870
$-0.1504210$

0.1206640

0.1291650

0.9424000

0.3459770

$-0.0265290$

$-0.6351710$

1. 0850310

0.5308190

$-0.9045440$

0.9820320

1. 9791140

1.0565590

$-0.5443460$

$-0.2344670$

$-0.6376960$

0.0649440

1. 9028450

1. 9335150

1.0628690

1.0768420

2.6892000

$-1.6500330$

$-3.2256560$

$-3.7301530$
0.2877360

$-1.1530680$

$-1.3431400$

$-0.2413130$

1. 1445240

1. 3107710

$-1.8019190$

$-1.4724740$

$-2.3336770$

$-1.3166700$

$-0.4125590$

$-0.2760420$

1.9320100

1.3090730

2. 3183810

0.5799210

$-0.2819240$

$-1.2879770$

$-0.2175040$

0.3914210

1.4598830

0.3713470

0.2452650

$-0.2423560$

0.3235830

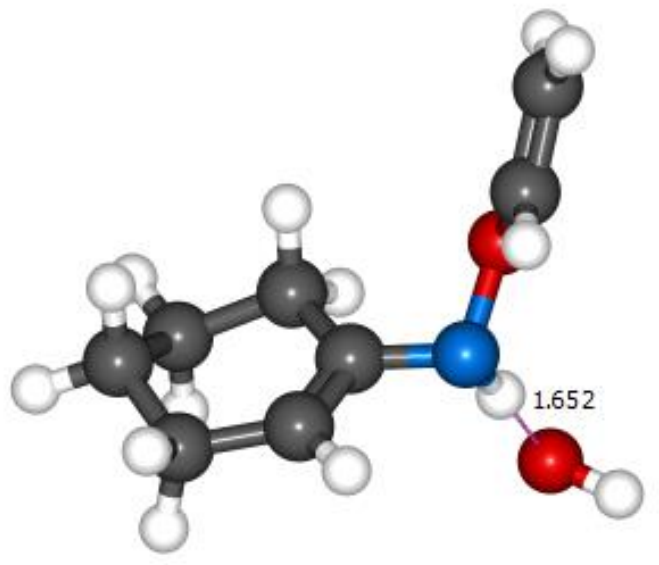

Complex of $O$-vinylhydroxylamine and hydroxide ion 9

$E$ (B2PLYP)

a.u.

$E$ (B2PLYP) $+Z P E$

$-518.0856504$

$E(\mathrm{~B} 2 \mathrm{PLYP})+H_{\text {corr }}$

$E(\mathrm{~B} 2 \mathrm{PLYP})+G_{\text {corr }}$

$-517.8766964$

$-517.8624444$

$-517.9183294$
0.0363430

$-0.3950470$

$-1.9027550$

$-2.7150270$

$-2.3510460$

$-0.8727300$

0.1896190

$-0.1282860$

$-2.1966300$

$-2.1266830$

$-3.7912460$

$-2.5050450$

$-2.7468100$

$-2.8371350$

$-0.5123610$

1. 3528400

1.9706810

$-0.2631970$

0.2567870

1. 4308740

1.9376510

$-0.1390920$

1.9605280

2. 9005190

3. 7948070
$-0.3476290$

$-1.3401390$

$-1.6363930$

$-0.3368340$

0.4528760

0.4914270

$-2.2603650$

$-0.9287760$

$-2.2173970$

$-2.2601050$

$-0.5488170$

0.2783750

1. 4760860

$-0.0044450$

1.1529830

$-0.1813720$

1. 0053680

2. 0158990

2. 6018310

2.1759320

2.7907880

3.5839410

$-0.9591510$

$-2.3658800$

$-2.3317380$
0.4765940

$-0.5911190$

$-0.5431250$

$-0.4585580$

0.8088510

1.0714750

$-0.4780460$

$-1.5767740$

$-1.4262930$

0.3348960

$-0.4672300$

$-1.3449840$

0.7628130

1. 6877270

1. 8549800

0.8094370

$-0.4657210$

$-1.4763890$

$-0.7246630$

$-0.1909620$

0.5655250

$-0.4780370$

0.4280000

0.0226170

0.4016200

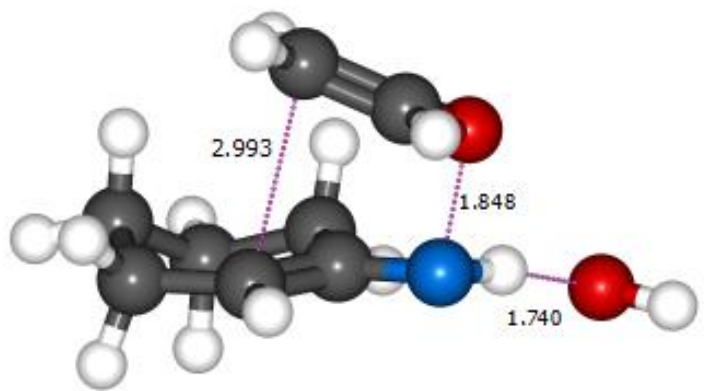

Transition state of [3.3]-sigmatropic shift

$\left(\mathrm{TS}_{9 \rightarrow 10}\right.$ )

$1267 \mathrm{~cm}^{-1}$

a.u.

$E$ (B2PLYP)

$-518.0650901$

$E$ (B2PLYP) $+Z P E$

$-517.8572591$

$E(\mathrm{~B} 2 \mathrm{PLYP})+H_{\text {corr }}$

$-517.8435941$

$E(\mathrm{~B} 2 \mathrm{PLYP})+G_{\text {corr }}$

$-517.8960311$ 

0.5362470
1.0654680
0.8526430
$-0.6116050$
$-1.0855780$
$-0.8890080$
2. 1224810
0.5268170
1.1733390
1.4945880
$-0.7285380$
$-1.2441480$
$-2.1405460$
$-0.5109630$
$-1.0332200$
1.1962260
$-1.8426170$
$-1.8254570$
$-1.9335570$
$-1.8998760$
$-1.9459180$
$-2.9352030$
2.1520400
3.8253930
4.4505330
0.4041210
$-0.8162870$
$-0.7010240$
$-0.3914150$
0.8825470
0.8272020
$-0.9546790$
$-1.7064070$
$-1.6308900$
0.1002470
$-0.2661570$
$-1.2447200$
1.0863240
1.7367980
1.8350090
1.0722000
$-0.9515990$
$-1.1507010$
$-0.1016910$
0.0000370
1.0291690
0.2325730
0.6720510
$-0.0719630$
0.3235420
1.3777380
2.0082540

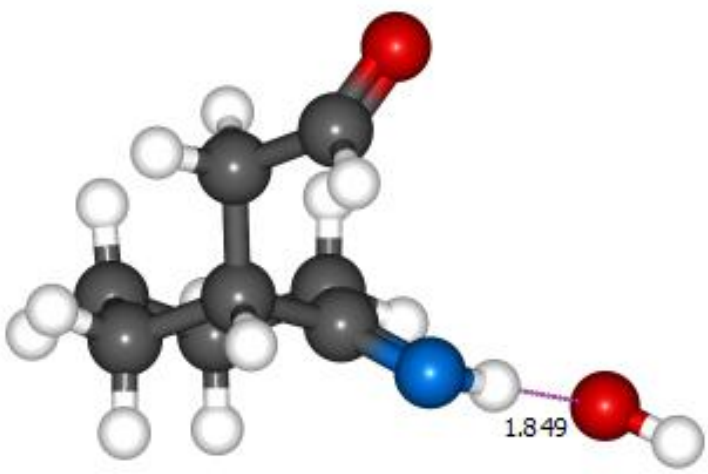

Complex of iminoaldehyde and hydroxide ion 10

a.u.

$E$ (B2PLYP)

$E(\mathrm{~B} 2 \mathrm{PLYP})+Z P E$

$E$ (B2PLYP) $+H_{\text {corr }}$

$E(\mathrm{~B} 2 \mathrm{PLYP})+G_{\text {corr }}$
$-518.1660112$

$-517.9544852$

$-517.9403472$

$-517.9953092$
$-0.1234860$

$-1.3734220$

$-2.7027120$

$-2.5585540$

$-1.5990400$

$-0.2253370$

$-1.3758570$

$-1.2523760$

$-3.4911790$

$-3.0049070$

$-3.5310620$

$-2.1864510$

$-1.4321900$

$-2.0968720$

$-0.0426130$

1. 0472870

3. 2831900

0.8420400

0.9319240

2. 3141650

2.4526080

0.8747140

1.0716240

1.1888400

2.0840410
0.4875090

1. 3124010

0.5511170

$-0.8295680$

$-1.6933360$

$-1.0242450$

2.1998000

1.6893160

1.1378280

0.4288750

$-1.3334680$

$-0.7159110$

$-2.6539520$

$-1.9303330$

$-1.1317290$

0.9956880

$-1.2957980$

$-1.6691440$

$-1.7486870$

$-1.3121010$

$-1.1293750$

$-2.8197600$

2.0335780

3. 8284380

4.2048030
$-0.2600350$

0.0482260

$-0.0415140$

0.6038730

$-0.2242610$

$-0.5309040$

$-0.5961870$

1.0741150

0.4461810

$-1.0920790$

0.6736440

1.6335360

0.2788860

$-1.1738920$

$-1.6104250$

$-0.2974110$

0.5221700

1. 2777020

0.1877180

$-0.2289390$

$-1.3108700$

$-0.0667580$

$-0.1321340$

0.1351640

0.0964980

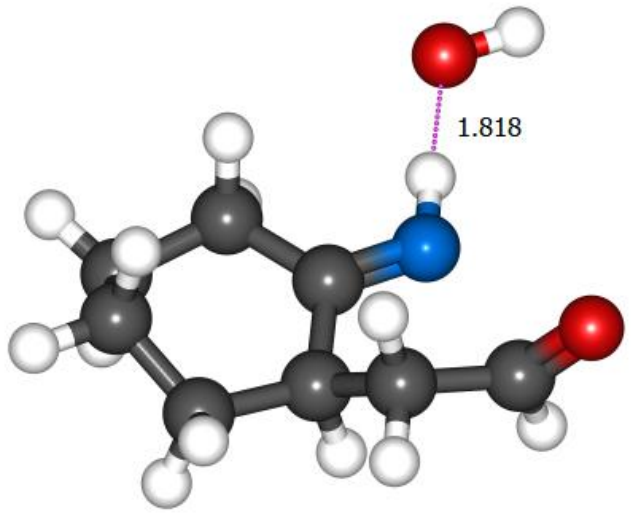

Transition state of conformation change of iminoaldehyde $\left(\mathrm{TS}_{10 \rightarrow 11}\right)$

$154 \mathrm{~cm}^{-1}$

a.u.

$E$ (B2PLYP)

$-518.157961$

$-517.946707$

$-517.933647$

$E(\mathrm{~B} 2 \mathrm{PLYP})+H_{\text {corr }}$

$-517.985489$ 


$\begin{array}{ll}\mathrm{C} & 0.1865330 \\ \mathrm{C} & 1.6217760 \\ \mathrm{C} & 2.6154660 \\ \mathrm{C} & 2.5575290 \\ \mathrm{C} & 1.1611840 \\ \mathrm{C} & 0.0252760 \\ \mathrm{H} & 1.6959200 \\ \mathrm{H} & 1.8558710 \\ \mathrm{H} & 3.6328040 \\ \mathrm{H} & 2.3813390 \\ \mathrm{H} & 2.8388190 \\ \mathrm{H} & 3.3007230 \\ \mathrm{H} & 1.1071050 \\ \mathrm{H} & 1.0022340 \\ \mathrm{H} & 0.1266200 \\ \mathrm{~N} & -0.8481720 \\ \mathrm{O} & -3.6347670 \\ \mathrm{H} & -1.5070800 \\ \mathrm{C} & -1.3660150 \\ \mathrm{C} & -2.5156280 \\ \mathrm{H} & -2.3354570 \\ \mathrm{H} & -1.4549700 \\ \mathrm{H} & -0.6175050 \\ \mathrm{O} & -0.2173950 \\ \mathrm{H} & -0.9886420\end{array}$

0.4661700

0.9526530

0.0272240

$-1.4198000$

$-1.7930930$

$-1.0432670$

1.9879440

0.9691720

0.4259940

0.0284490

$-2.1185350$

$-1.5462260$

$-1.5522540$

$-2.8755340$

$-1.2494040$

1.2133210

$-0.7172020$

$-1.3968970$

$-1.5166670$

$-0.8525360$

$-0.5811340$

$-2.5931490$

2.2359400

3.9993570

4.5910380
0.0021920

$-0.1604840$

0.5596690

0.0109580

$-0.5439840$

0.1785780

0.1885860

$-1.2367200$

0.4686190

1. 6328400

0.8090080

$-0.7864120$

$-1.6148100$

$-0.4649480$

1. 2581350

0.0044870

$-0.0268140$

$-1.3443240$

$-0.2634140$

0.4528390

1.5105710

$-0.0437500$

$-0.0798460$

$-0.1707180$

$-0.1662260$

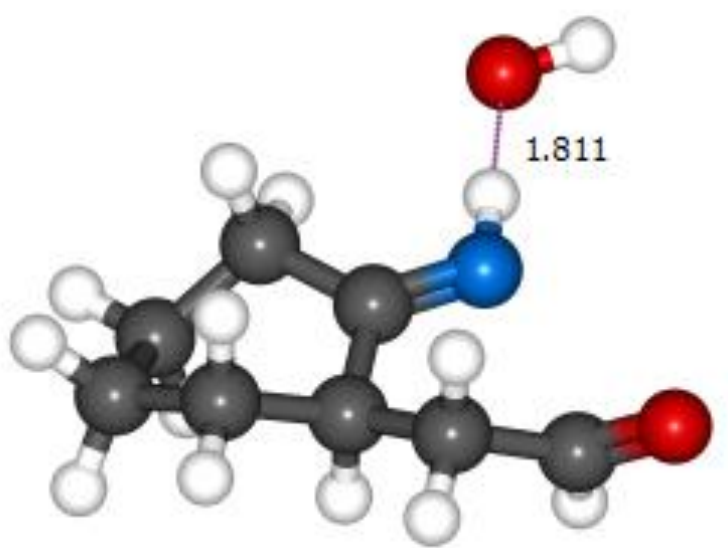

Complex of iminoaldehyde and hydroxide ion 11

E(B2PLYP)

a.u.

$E$ (B2PLYP) $+Z P E$

$-518.164455$

$E$ (B2PLYP) $+H_{\text {corr }}$

$E(\mathrm{~B} 2 \mathrm{PLYP})+G_{\text {corr }}$
$-517.952533$

$-517.93899$

$-517.99152$

\begin{tabular}{llll}
\hline & & & \\
$\mathrm{C}$ & 0.0698140 & 0.4253630 & 0.0785920 \\
$\mathrm{C}$ & 1.0841720 & 1.5300410 & -0.1372750 \\
$\mathrm{C}$ & 2.4482360 & 1.1366010 & 0.4561050 \\
$\mathrm{C}$ & 3.0002570 & -0.1787740 & -0.1457950 \\
$\mathrm{C}$ & 1.8903250 & -1.1985530 & -0.5180040 \\
$\mathrm{C}$ & 0.6044370 & -0.9825460 & 0.2968250 \\
$\mathrm{H}$ & 0.7149170 & 2.4710240 & 0.2847740 \\
$\mathrm{H}$ & 1.1821300 & 1.6918690 & -1.2217530 \\
$\mathrm{H}$ & 3.1674520 & 1.9495120 & 0.3028860 \\
$\mathrm{H}$ & 2.3359770 & 1.0279630 & 1.5431200 \\
$\mathrm{H}$ & 3.6957510 & -0.6286470 & 0.5734950 \\
$\mathrm{H}$ & 3.5891490 & 0.0462000 & -1.0438650 \\
$\mathrm{H}$ & 1.6377970 & -1.1029550 & -1.5830140 \\
$\mathrm{H}$ & 2.2538110 & -2.2239730 & -0.3798150 \\
$\mathrm{H}$ & 0.8532810 & -1.0753450 & 1.3670220 \\
$\mathrm{~N}$ & -1.1976520 & 0.5286030 & 0.0803160 \\
$\mathrm{O}$ & -2.9678260 & -1.5688920 & -0.2211010 \\
$\mathrm{H}$ & -0.6135580 & -2.0985640 & -1.1183500 \\
$\mathrm{C}$ & -0.5631230 & -1.9203080 & -0.0359630 \\
$\mathrm{C}$ & -1.9025800 & -1.3182020 & 0.4015310 \\
$\mathrm{H}$ & -1.9628750 & -1.1293920 & 1.4921760 \\
$\mathrm{H}$ & -0.4415420 & -2.8935350 & 0.4588860 \\
$\mathrm{H}$ & -1.6807880 & 1.4898100 & -0.0399910 \\
$\mathrm{O}$ & -2.3809820 & 2.9387000 & -0.2080750 \\
$\mathrm{H}$ & -3.3466980 & 2.8556120 & -0.1404730
\end{tabular}

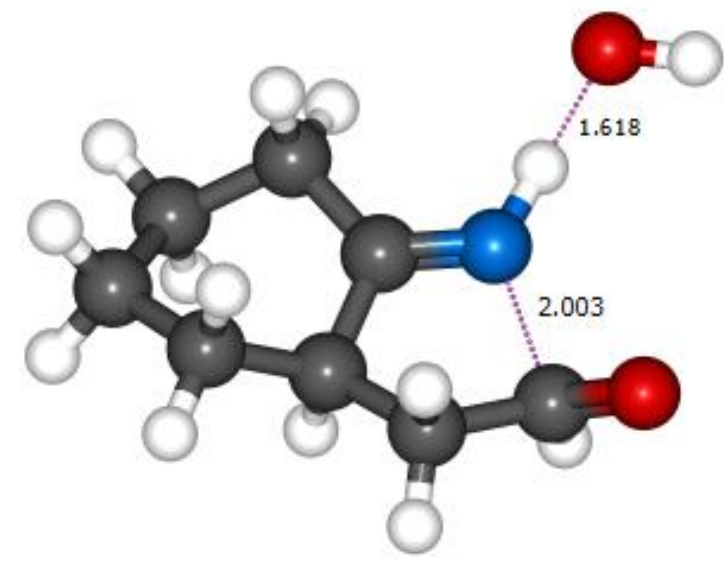

Transition state of iminoaldehyde cyclization

$\left(\mathrm{TS}_{11 \rightarrow 12}\right)$

$I 161 \mathrm{~cm}^{-1}$

$E$ (B2PLYP)

$E(\mathrm{~B} 2 \mathrm{PLYP})+Z P E$

$E$ (B2PLYP $)+H_{\text {corr }}$

$E(\mathrm{~B} 2 \mathrm{PLYP})+G_{\text {corr }}$ a.u.

$-518.1589638$

$-517.9479408$

$-517.9354488$

$-517.9855818$ 


\begin{tabular}{llll}
\hline & & & \\
$\mathrm{C}$ & 0.0884600 & 0.3594800 & 0.1156030 \\
$\mathrm{C}$ & 0.9162200 & 1.5958000 & -0.1481020 \\
$\mathrm{C}$ & 2.3464690 & 1.3989780 & 0.3845160 \\
$\mathrm{C}$ & 3.0467070 & 0.1640420 & -0.2347160 \\
$\mathrm{C}$ & 2.0877620 & -1.0355180 & -0.4908370 \\
$\mathrm{C}$ & 0.8158620 & -0.9538910 & 0.3670550 \\
$\mathrm{H}$ & 0.4399220 & 2.4802020 & 0.2919420 \\
$\mathrm{H}$ & 0.9480220 & 1.7681300 & -1.2352950 \\
$\mathrm{H}$ & 2.9445010 & 2.2983840 & 0.1953700 \\
$\mathrm{H}$ & 2.2984700 & 1.2858780 & 1.4759700 \\
$\mathrm{H}$ & 3.8627940 & -0.1438610 & 0.4309880 \\
$\mathrm{H}$ & 3.5175590 & 0.4509950 & -1.1839160 \\
$\mathrm{H}$ & 1.7834510 & -1.0483530 & -1.5466400 \\
$\mathrm{H}$ & 2.6095210 & -1.9838610 & -0.3109720 \\
$\mathrm{H}$ & 1.1083760 & -0.9823980 & 1.4303450 \\
$\mathrm{~N}$ & -1.1882030 & 0.2942980 & 0.1212900 \\
$\mathrm{O}$ & -2.7004640 & -1.5216420 & -0.3713170 \\
$\mathrm{H}$ & -0.3131750 & -2.2749910 & -0.9432720 \\
$\mathrm{C}$ & -0.3143560 & -1.9610640 & 0.1105100 \\
$\mathrm{C}$ & -1.6361960 & -1.1594080 & 0.3608740 \\
$\mathrm{H}$ & -1.8386800 & -1.1746140 & 1.4655130 \\
$\mathrm{H}$ & -0.2515780 & -2.8597280 & 0.7353780 \\
$\mathrm{H}$ & -2.4234840 & 1.5829060 & -0.0512140 \\
$\mathrm{O}$ & -3.1172740 & 2.3033430 & -0.1308280 \\
$\mathrm{H}$ & -3.9319400 & 1.8371020 & -0.3754820
\end{tabular}

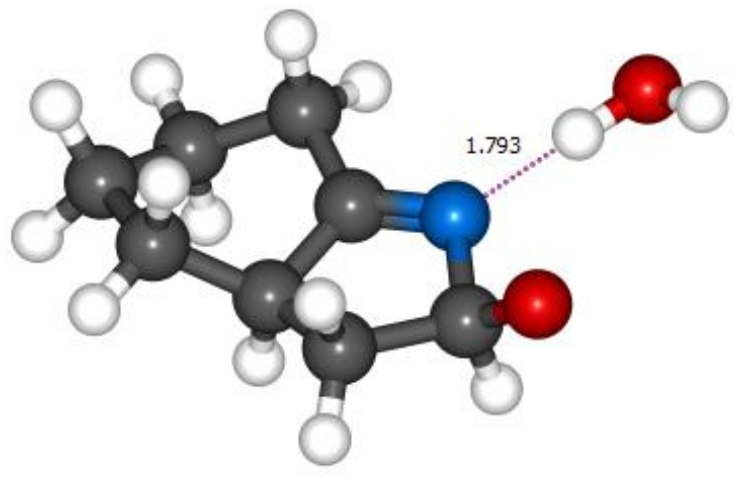
Complex of 5-hydroxypyrroline anion and
water molecule 12

$E$ (B2PLYP)

a.u.

$E(\mathrm{~B} 2 \mathrm{PLYP})+Z P E$

$-518.1900587$

$E(\mathrm{~B} 2 \mathrm{PLYP})+H_{\text {corr }}$

$-517.9767617$

$E(\mathrm{~B} 2 \mathrm{PLYP})+G_{\text {corr }}$

$-518.0158407$

\begin{tabular}{|c|c|c|c|c|}
\hline $\mathrm{C}$ & 0.4805260 & 0.6378180 & -0.0272830 & \\
\hline C & 1.6287690 & 1.5739930 & -0.3246800 & \\
\hline C & 2.9100580 & 1.0801320 & 0.3709560 & \\
\hline $\mathrm{C}$ & 3.2967010 & -0.3608720 & -0.0465840 & \\
\hline $\mathrm{C}$ & 2.0724140 & -1.2890060 & -0.2949380 & \\
\hline C & 0.8113070 & -0.7815790 & 0.4197950 & \\
\hline $\mathrm{H}$ & 1.3774880 & 2.5990330 & -0.0262730 & \\
\hline $\mathrm{H}$ & 1.7879030 & 1.5967950 & -1.4142380 & \\
\hline $\mathrm{H}$ & 3.7409050 & 1.7634140 & 0.1580000 & \\
\hline $\mathrm{H}$ & 2.7527120 & 1.1178840 & 1.4574810 & \\
\hline $\mathrm{H}$ & 3.9393400 & -0.7888830 & 0.7331600 & ypyrroline anio \\
\hline $\mathrm{H}$ & 3.9057980 & -0.3248010 & -0.9591780 & water molecule 13 \\
\hline $\mathrm{H}$ & 1.8553020 & -1.3365580 & -1.3712230 & a.u. \\
\hline $\mathrm{H}$ & 2.3042980 & -2.3152440 & 0.0168470 & -518.1960485 \\
\hline $\mathrm{H}$ & 1.0058090 & -0.7638440 & 1.5056520 & $E(\mathrm{~B} 2 \mathrm{PLYP})+Z P E$ \\
\hline N & -0.7627430 & 0.9128300 & -0.1484650 & $\begin{array}{l}E(\mathrm{~B} 2 \mathrm{PLYP})+\angle P E \\
E(\mathrm{~B} 2 \mathrm{PLY})+H_{\text {corr }}\end{array}$ \\
\hline $\begin{array}{l}\mathrm{O} \\
\mathrm{H}\end{array}$ & $\begin{array}{l}-2.6621880 \\
-0.5392520\end{array}$ & -1.8933550 & -0.8747170 & $E(\mathrm{~B} 2 \mathrm{PLYP})+G_{c o r r}$ \\
\hline $\mathrm{C}$ & -0.5283240 & -1.4782200 & 0.1433630 & \\
\hline $\mathrm{C}$ & -1.5780630 & -0.3266800 & 0.1939450 & \\
\hline $\mathrm{H}$ & -1.8709580 & -0.1644640 & 1.2604750 & \\
\hline $\mathrm{H}$ & -0.7608160 & -2.2869100 & 0.8458680 & \\
\hline O & -4.7755900 & 0.7252690 & 0.1783540 & \\
\hline $\mathrm{H}$ & -3.9095420 & 0.2303810 & -0.1245560 & \\
\hline $\mathrm{H}$ & -5.3078870 & 0.0616830 & 0.6431230 & \\
\hline
\end{tabular}




\begin{tabular}{llll}
\hline & & & \\
$\mathrm{C}$ & -1.0418030 & 0.3181920 & -0.4862830 \\
$\mathrm{C}$ & -2.2191070 & 1.0970300 & -1.0243380 \\
$\mathrm{C}$ & -3.4387850 & 0.1722160 & -1.1862030 \\
$\mathrm{C}$ & -3.8384080 & -0.5257370 & 0.1378050 \\
$\mathrm{C}$ & -2.6250560 & -0.9022830 & 1.0367910 \\
$\mathrm{C}$ & -1.3191300 & -0.9958750 & 0.2335890 \\
$\mathrm{H}$ & -1.9564870 & 1.5867330 & -1.9698960 \\
$\mathrm{H}$ & -2.4589560 & 1.9027100 & -0.3127460 \\
$\mathrm{H}$ & -4.2910660 & 0.7402020 & -1.5778920 \\
$\mathrm{H}$ & -3.2015390 & -0.5864350 & -1.9442560 \\
$\mathrm{H}$ & -4.4190580 & -1.4248110 & -0.1041160 \\
$\mathrm{H}$ & -4.5111160 & 0.1294330 & 0.7062710 \\
$\mathrm{H}$ & -2.4891890 & -0.1386880 & 1.8153690 \\
$\mathrm{H}$ & -2.8198860 & -1.8452470 & 1.5628810 \\
$\mathrm{H}$ & -1.4292190 & -1.7878200 & -0.5265800 \\
$\mathrm{~N}$ & 0.1845060 & 0.6763950 & -0.5483320 \\
$\mathrm{O}$ & 2.0760340 & 0.1622340 & 0.8598890 \\
$\mathrm{H}$ & -0.0603200 & -0.7237290 & 1.9883860 \\
$\mathrm{C}$ & 0.0030690 & -1.1825020 & 0.9912610 \\
$\mathrm{C}$ & 1.0377720 & -0.3675690 & 0.1555310 \\
$\mathrm{H}$ & 1.4056760 & -1.0182170 & -0.6763030 \\
$\mathrm{H}$ & 0.2990960 & -2.2306070 & 1.1145250 \\
$\mathrm{O}$ & 1.9703850 & 2.7932480 & 0.8976960 \\
$\mathrm{H}$ & 2.0286770 & 1.7756970 & 0.9050310 \\
$\mathrm{H}$ & 1.2572630 & 2.9415110 & 0.2554400 \\
$\mathrm{C}$ & 4.4772680 & -0.7559580 & -0.4767540 \\
$\mathrm{H}$ & 3.5755530 & -0.3823040 & 0.0459430 \\
$\mathrm{C}$ & 5.4737170 & -1.1582220 & -1.0419220 \\
$\mathrm{H}$ & 6.3504520 & -1.5128100 & -1.5412770 \\
& & &
\end{tabular}

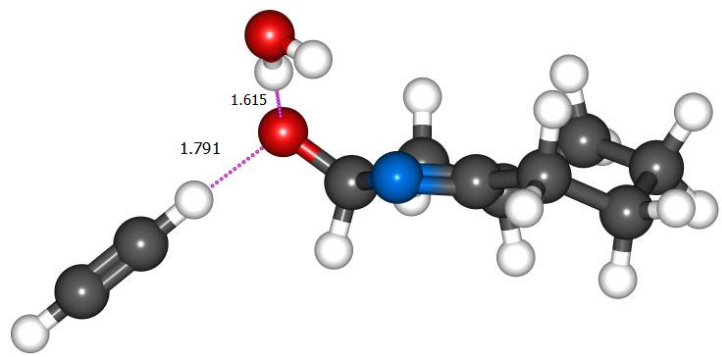

Complex of 5-hydroxypyrroline anion, acetylene and water molecule 14

a.u.

$E$ (B2PLYP)

$-595.5014346$

$E(\mathrm{~B} 2 \mathrm{PLYP})+Z P E$

$-595.2594326$

$E(\mathrm{~B} 2 \mathrm{PLYP})+H_{\text {corr }}$

$-595.2432516$

$E(\mathrm{~B} 2 \mathrm{PLYP})+G_{\text {corr }}$

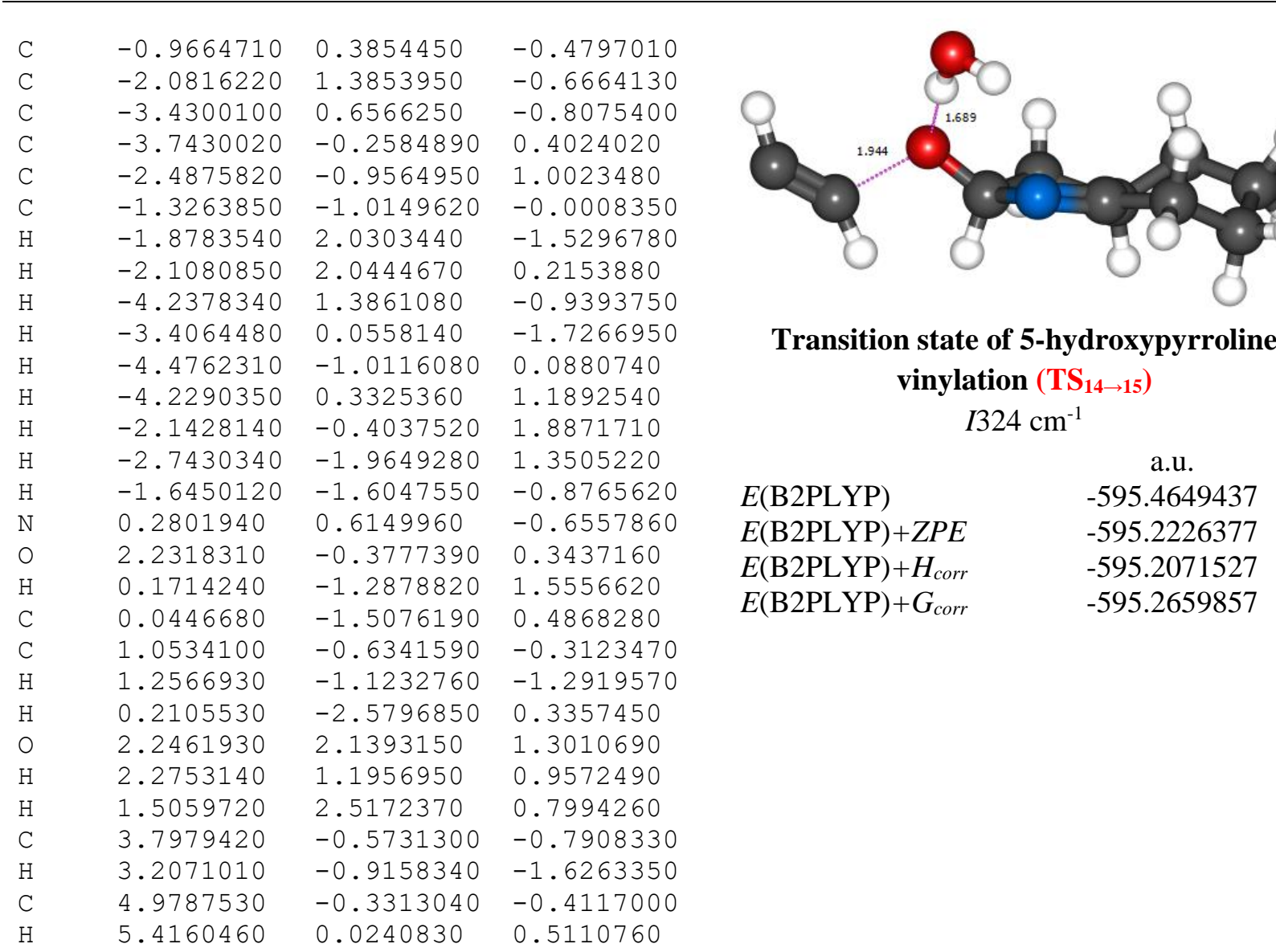




$\begin{array}{llll}\mathrm{C} & -0.8960600 & 0.1857760 & -0.6811710 \\ \mathrm{C} & -1.9942460 & 1.1483290 & -1.0505910 \\ \mathrm{C} & -3.3687370 & 0.4828250 & -0.8594760 \\ \mathrm{C} & -3.5838850 & -0.0407700 & 0.5822420 \\ \mathrm{C} & -2.2959400 & -0.6157270 & 1.2413960 \\ \mathrm{C} & -1.2427060 & -1.0082110 & 0.1944970 \\ \mathrm{H} & -1.8575320 & 1.5107000 & -2.0761840 \\ \mathrm{H} & -1.9132380 & 2.0295210 & -0.3954940 \\ \mathrm{H} & -4.1642820 & 1.1906830 & -1.1190610 \\ \mathrm{H} & -3.4546360 & -0.3497950 & -1.5704100 \\ \mathrm{H} & -4.3672060 & -0.8081520 & 0.5596170 \\ \mathrm{H} & -3.9685250 & 0.7720180 & 1.2110990 \\ \mathrm{H} & -1.8497660 & 0.1368020 & 1.9056810 \\ \mathrm{H} & -2.5429400 & -1.4769990 & 1.8736030 \\ \mathrm{H} & -1.6598690 & -1.7982590 & -0.4505580 \\ \mathrm{~N} & 0.3387840 & 0.3018520 & -1.0061760 \\ \mathrm{O} & 2.3095700 & -0.3962950 & 0.1692570 \\ \mathrm{H} & 0.3825810 & -0.9415880 & 1.6441330 \\ \mathrm{C} & 0.1579660 & -1.4202610 & 0.6824940 \\ \mathrm{C} & 1.0850250 & -0.8253510 & -0.3970480 \\ \mathrm{H} & 1.3019320 & -1.5540650 & -1.1918460 \\ \mathrm{H} & 0.2883710 & -2.5001030 & 0.7983660 \\ \mathrm{O} & 1.9396660 & 2.0801360 & 1.4659880 \\ \mathrm{H} & 2.0964930 & 1.2124880 & 1.0241980 \\ \mathrm{H} & 1.3090960 & 2.5300590 & 0.8820180 \\ \mathrm{C} & 3.4570590 & -0.3410000 & -0.7281180 \\ \mathrm{H} & 3.1222410 & -0.5396600 & -1.7489870 \\ \mathrm{C} & 4.7075340 & -0.0799670 & -0.3406400 \\ \mathrm{H} & 4.7558510 & 0.0888080 & 0.7535720\end{array}$

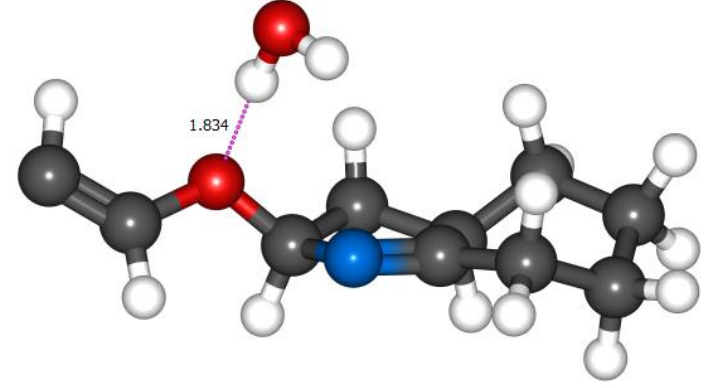

Complex of 5-vinyloxypyrroline anion and water molecule 15

$E$ (B2PLYP)

a.u.

$E(\mathrm{~B} 2 \mathrm{PLYP})+Z P E$

$-595.4612445$

$E(\mathrm{~B} 2 \mathrm{PLYP})+H_{\text {corr }}$

$E(\mathrm{~B} 2 \mathrm{PLYP})+G_{\text {corr }}$

$-595.2154645$

$-595.1998805$

$-595.2587475$

\begin{tabular}{|c|c|c|c|c|c|}
\hline $\mathrm{C}$ & 1.1488710 & -0.6680930 & -0.6183660 & & \\
\hline $\mathrm{C}$ & 2.5248030 & -1.2208310 & -0.8892580 & & \\
\hline $\mathrm{C}$ & 3.6216500 & -0.3595880 & -0.2326460 & & \\
\hline $\mathrm{C}$ & 3.1753730 & 0.0895440 & 1.1664630 & & \\
\hline C & 1.9828410 & 1.0699740 & 1.0956970 & & \\
\hline $\mathrm{C}$ & 0.9758030 & 0.7274840 & -0.0385400 & & \\
\hline $\mathrm{H}$ & 2.6778710 & -1.2916150 & -1.9748720 & & \\
\hline $\mathrm{H}$ & 2.5646800 & -2.2489690 & -0.5059040 & & \\
\hline $\mathrm{H}$ & 4.5541950 & -0.9333290 & -0.1803320 & & \\
\hline $\mathrm{H}$ & 3.8297270 & 0.5239980 & -0.8524620 & & \\
\hline $\mathrm{H}$ & 4.0052180 & 0.5613780 & 1.7064420 & & \\
\hline $\mathrm{H}$ & 2.8921620 & -0.8013690 & 1.7465540 & & \\
\hline $\mathrm{H}$ & 1.4593630 & 1.0780330 & 2.0595440 & \multirow{3}{*}{\multicolumn{2}{|c|}{$\begin{array}{c}\text { Complex of 5-vinyloxypyrroline and } \\
\text { hydroxide anion } 16\end{array}$}} \\
\hline $\mathrm{H}$ & 2.3607560 & 2.0874980 & 0.9398710 & & \\
\hline $\mathrm{H}$ & 1.1434240 & 1.4293410 & -0.8701140 & & \\
\hline $\mathrm{N}$ & 0.0645230 & -1.3080230 & -0.8703380 & & a.u. \\
\hline O & -2.0411690 & -1.2528370 & 0.1525150 & $E(\mathrm{~B} 2 \mathrm{PLYP})$ & -595.4874536 \\
\hline $\mathrm{H}$ & -0.6578340 & 0.4568560 & 1.3783160 & $E(\mathrm{~B} 2 \mathrm{PLYP})+Z P E$ & -595.2417686 \\
\hline $\mathrm{C}$ & -0.5259440 & 0.7150120 & 0.3198830 & $E(\mathrm{~B} 2 \mathrm{PLYP})+H_{\text {corr }}$ & -595.2257376 \\
\hline $\mathrm{C}$ & -1.0692880 & -0.4475750 & -0.5366670 & $E(\mathrm{~B} 2 \mathrm{PLYP})+G_{\text {corr }}$ & -595.2882056 \\
\hline $\mathrm{H}$ & -1.5290470 & -0.0963480 & -1.4711800 & & \\
\hline $\mathrm{H}$ & -1.0348570 & 1.6630790 & 0.1181420 & & \\
\hline O & -2.0150920 & 3.6767100 & -0.6005650 & & \\
\hline $\mathrm{H}$ & -2.4039030 & 4.4952340 & -0.9540400 & & \\
\hline $\mathrm{C}$ & -3.3414130 & -0.8526070 & 0.0621280 & & \\
\hline $\mathrm{H}$ & -3.5431620 & -0.0959530 & -0.6961870 & & \\
\hline C & -4.3074180 & -1.3700200 & 0.8299090 & & \\
\hline $\mathrm{H}$ & -4.0995610 & -2.1191700 & 1.5888510 & & \\
\hline $\mathrm{H}$ & -5.3322750 & -1.0432970 & 0.6925160 & & \\
\hline
\end{tabular}




$\begin{array}{llll}\mathrm{C} & 1.0094670 & 0.1160950 & -0.8137020 \\ \mathrm{C} & 2.3176710 & -0.3891270 & -1.3580060 \\ \mathrm{C} & 3.2092080 & -0.9004550 & -0.2016160 \\ \mathrm{C} & 2.3694560 & -1.6599300 & 0.8420950 \\ \mathrm{C} & 1.2817060 & -0.7671400 & 1.5080780 \\ \mathrm{C} & 0.9118890 & 0.4838440 & 0.6452010 \\ \mathrm{H} & 2.8305770 & 0.4143610 & -1.9066420 \\ \mathrm{H} & 2.1222350 & -1.1881540 & -2.0848590 \\ \mathrm{H} & 3.9960820 & -1.5494450 & -0.6044150 \\ \mathrm{H} & 3.7186580 & -0.0536040 & 0.2780210 \\ \mathrm{H} & 3.0255550 & -2.0938570 & 1.6071110 \\ \mathrm{H} & 1.8846520 & -2.5075480 & 0.3362200 \\ \mathrm{H} & 0.3764110 & -1.3590030 & 1.6839660 \\ \mathrm{H} & 1.6265960 & -0.4192210 & 2.4900460 \\ \mathrm{H} & 1.6928280 & 1.2369080 & 0.8509960 \\ \mathrm{~N} & -0.0897580 & 0.2134160 & -1.4809940 \\ \mathrm{O} & -1.9716400 & -0.8317440 & -0.3895500 \\ \mathrm{H} & -1.0606810 & 1.0095800 & 1.6184680 \\ \mathrm{C} & -0.4894590 & 1.0808260 & 0.6883030 \\ \mathrm{C} & -1.1263290 & 0.6082010 & -0.5312130 \\ \mathrm{H} & -1.9302760 & 1.1925060 & -0.9886500 \\ \mathrm{H} & -0.3371930 & 2.7402000 & 0.5164490 \\ \mathrm{O} & -0.2372420 & 3.8153780 & 0.4748390 \\ \mathrm{H} & 0.1854780 & 3.9977840 & -0.3807790 \\ \mathrm{C} & -3.2670520 & -0.6958770 & -0.1106850 \\ \mathrm{H} & -3.6681380 & 0.3200270 & -0.2173620 \\ \mathrm{C} & -4.0951590 & -1.6936080 & 0.2714460 \\ \mathrm{H} & -3.7472210 & -2.7170630 & 0.3914650 \\ \mathrm{H} & -5.1445860 & -1.4834240 & 0.4552130\end{array}$

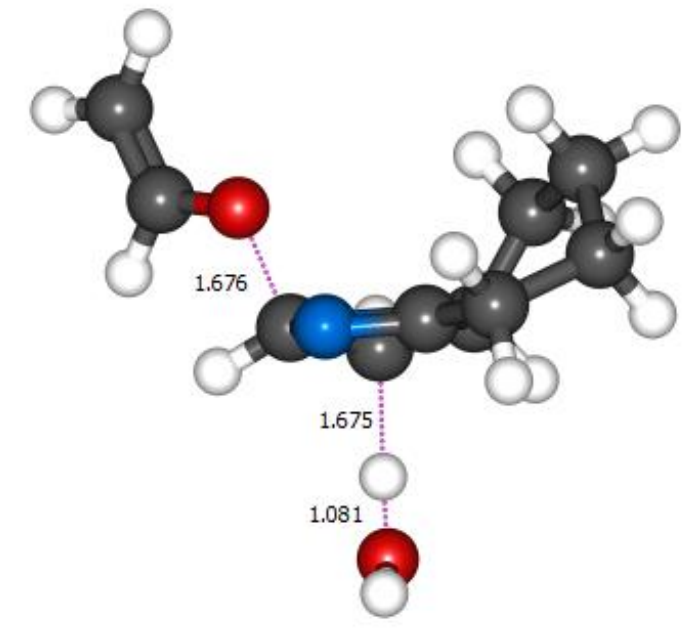

Transition state of $\mathbf{3 H}$-pyrrole formation from 5 -vinyloxypyrroline $\left(\mathrm{TS}_{16 \rightarrow 17}\right)$

$1383 \mathrm{~cm}^{-1}$

a.u.

$E$ (B2PLYP)

$E(\mathrm{~B} 2 \mathrm{PLYP})+Z P E$

$E(\mathrm{~B} 2 \mathrm{PLYP})+H_{\text {corr }}$

$E(\mathrm{~B} 2 \mathrm{PLYP})+G_{\text {corr }}$

$-595.4858802$

$-595.2452702$

$-595.2300362$

$-595.2885522$

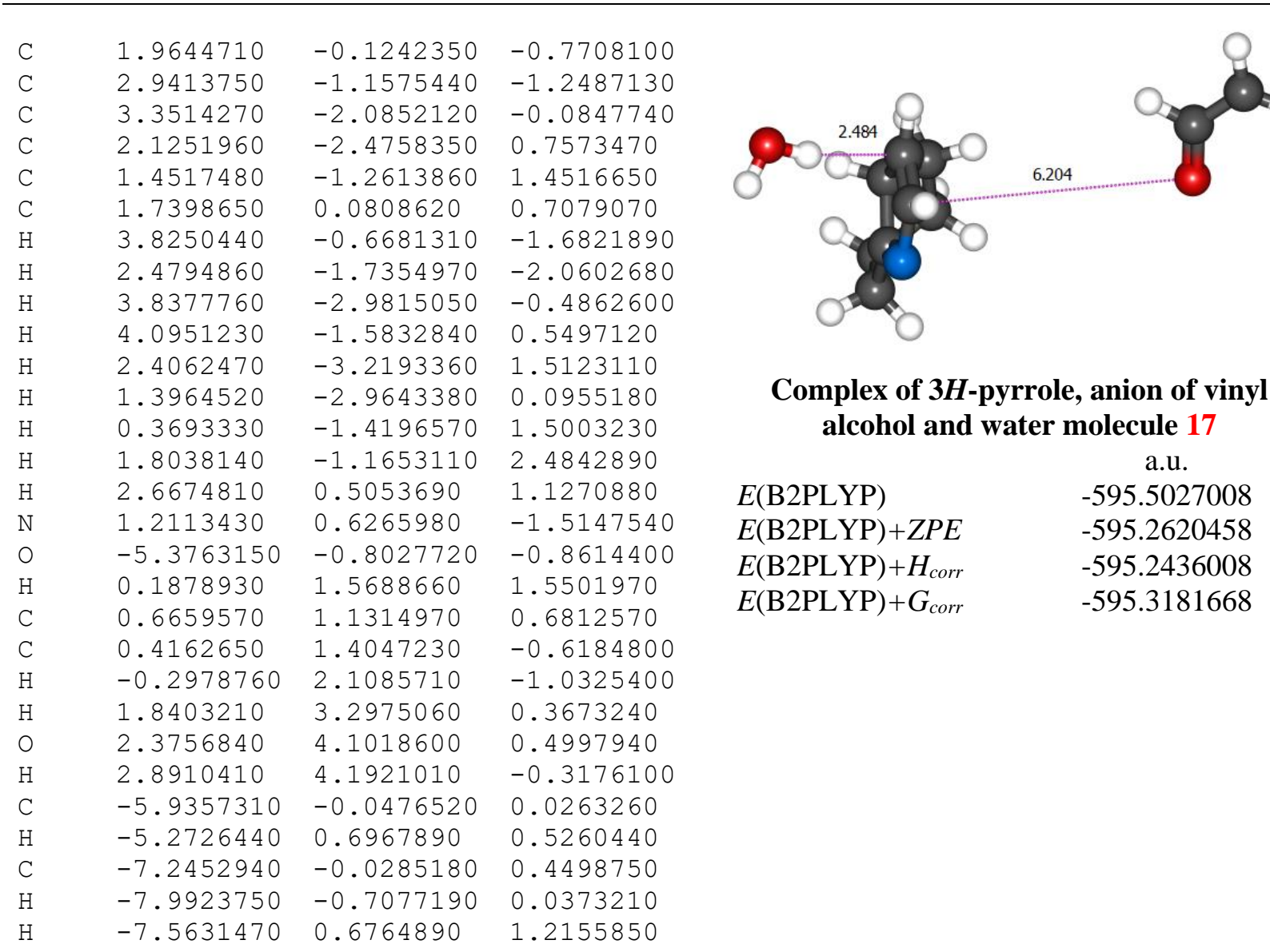




$\begin{array}{llll}\mathrm{C} & 0.3538130 & -0.7750310 & 0.0714980 \\ \mathrm{C} & -0.9561990 & -1.5063410 & 0.0999240 \\ \mathrm{C} & -2.1185490 & -0.5145860 & 0.3190470 \\ \mathrm{C} & -1.9265610 & 0.7473810 & -0.5395610 \\ \mathrm{C} & -0.6582120 & 1.5549780 & -0.1545710 \\ \mathrm{C} & 0.4305460 & 0.6648650 & 0.5217260 \\ \mathrm{H} & -0.9453530 & -2.2680840 & 0.8923990 \\ \mathrm{H} & -1.0840210 & -2.0478710 & -0.8470560 \\ \mathrm{H} & -3.0677740 & -1.0029440 & 0.0707880 \\ \mathrm{H} & -2.1778150 & -0.2362240 & 1.3804760 \\ \mathrm{H} & -2.8089650 & 1.3938020 & -0.4658500 \\ \mathrm{H} & -1.8542400 & 0.4380820 & -1.5917890 \\ \mathrm{H} & -0.2353780 & 2.0223080 & -1.0503180 \\ \mathrm{H} & -0.9163560 & 2.3678840 & 0.5327630 \\ \mathrm{H} & 0.2494520 & 0.6771740 & 1.6097590 \\ \mathrm{~N} & 1.4875810 & -1.2397650 & -0.3549700 \\ \mathrm{C} & 1.8788090 & 0.9616730 & 0.2533790 \\ \mathrm{C} & 2.4289440 & -0.1705980 & -0.2316530 \\ \mathrm{H} & 3.4575290 & -0.3464720 & -0.5278180 \\ \mathrm{H} & 2.3743040 & 1.9066600 & 0.4427030\end{array}$

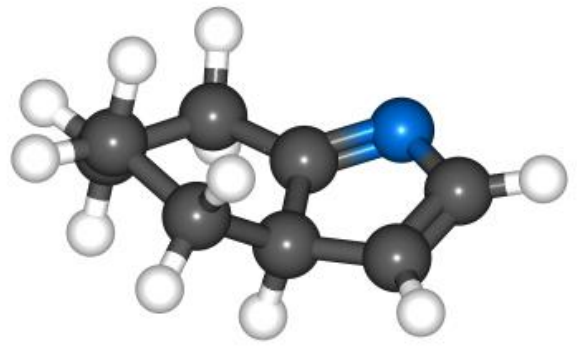

$3 H$-pyrrole 18

a.u.

$E$ (B2PLYP)

$-365.9478196$

$E(\mathrm{~B} 2 \mathrm{PLYP})+Z P E$

$-365.7728376$

$E(\mathrm{~B} 2 \mathrm{PLYP})+H_{\text {corr }}$

$E(\mathrm{~B} 2 \mathrm{PLYP})+G_{\text {corr }}$

$-365.7644036$

$-365.8052586$

$\begin{array}{llll}\mathrm{C} & -0.7467800 & 0.3420900 & -0.7589560 \\ \mathrm{C} & -1.9847190 & 0.8217350 & -1.4673070 \\ \mathrm{C} & -3.2349190 & 0.4908090 & -0.6330590 \\ \mathrm{C} & -3.1698790 & 1.0772190 & 0.7984150 \\ \mathrm{C} & -1.7488150 & 1.0318490 & 1.4345590 \\ \mathrm{C} & -0.8329040 & 0.0246180 & 0.7229980 \\ \mathrm{H} & -2.0410090 & 0.3907460 & -2.4736890 \\ \mathrm{H} & -1.9004700 & 1.9113650 & -1.5995710 \\ \mathrm{H} & -4.1306960 & 0.8614170 & -1.1442400 \\ \mathrm{H} & -3.3401880 & -0.6008310 & -0.5803290 \\ \mathrm{H} & -3.8801030 & 0.5298860 & 1.4296560 \\ \mathrm{H} & -3.5144070 & 2.1187620 & 0.7795900 \\ \mathrm{H} & -1.2792540 & 2.0226520 & 1.3694040 \\ \mathrm{H} & -1.8150600 & 0.7914280 & 2.5020990 \\ \mathrm{H} & -1.2601280 & -0.9849560 & 0.8320360 \\ \mathrm{~N} & 0.4113280 & 0.2101400 & -1.2961000 \\ \mathrm{O} & 2.5635910 & 0.4996950 & -0.4219390 \\ \mathrm{H} & 0.9747050 & 0.9826970 & 1.4792740 \\ \mathrm{C} & 0.6607390 & 0.0020720 & 1.0999960 \\ \mathrm{C} & 1.3454600 & -0.2431930 & -0.2633830 \\ \mathrm{H} & 1.5731750 & -1.3053130 & -0.4305300 \\ \mathrm{H} & 0.9171080 & -0.7552420 & 1.8459670 \\ \mathrm{O} & -1.2763630 & -4.1073080 & 0.1044460 \\ \mathrm{H} & -0.8113680 & -3.2798190 & -0.1060370 \\ \mathrm{C} & 3.7055930 & -0.0964270 & 0.0277650 \\ \mathrm{H} & 3.6282790 & -1.1682570 & 0.2117010 \\ \mathrm{C} & 4.8474490 & 0.5788210 & 0.1992720 \\ \mathrm{H} & 4.9154160 & 1.6481560 & 0.0197080 \\ \mathrm{H} & 5.7395280 & 0.0496880 & 0.5158150\end{array}$

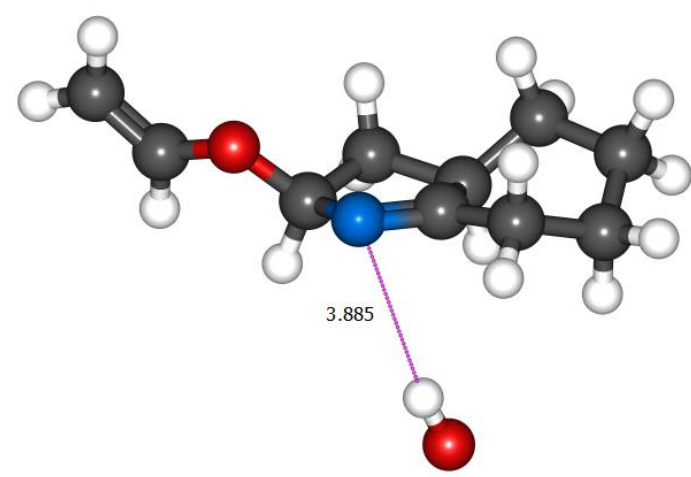

Complex of 5-vinyloxypyrroline and hydroxide anion 19

a.u.

$E$ (B2PLYP)

$-595.4806558$

$E(\mathrm{~B} 2 \mathrm{PLYP})+Z P E$

$E(\mathrm{~B} 2 \mathrm{PLYP})+H_{\text {corr }}$

$E(\mathrm{~B} 2 \mathrm{PLYP})+G_{\text {corr }}$

$-595.2347758$

$-595.2187898$

$-595.2812058$ 


$\begin{array}{llll} & & & \\ \mathrm{C} & -0.8640730 & -0.7320780 & -0.1028810 \\ \mathrm{C} & -2.0163070 & -0.8159870 & -1.1086910 \\ \mathrm{C} & -3.1695980 & 0.1509300 & -0.8118860 \\ \mathrm{C} & -2.6217680 & 1.5562460 & -0.5290180 \\ \mathrm{C} & -1.8430400 & 1.5664540 & 0.7951100 \\ \mathrm{C} & -0.7781100 & 0.4459340 & 0.9162320 \\ \mathrm{H} & -2.3832410 & -1.8446780 & -1.1928460 \\ \mathrm{H} & -1.5975610 & -0.5509300 & -2.0905810 \\ \mathrm{H} & -3.8588580 & 0.1671240 & -1.6669750 \\ \mathrm{H} & -3.7488270 & -0.1937910 & 0.0568920 \\ \mathrm{H} & -3.4362440 & 2.2914580 & -0.4786570 \\ \mathrm{H} & -1.9706420 & 1.8644500 & -1.3617880 \\ \mathrm{H} & -1.3673150 & 2.5454160 & 0.9475770 \\ \mathrm{H} & -2.5729300 & 1.4540050 & 1.6089300 \\ \mathrm{H} & -0.8696030 & 0.0002010 & 1.9106330 \\ \mathrm{~N} & 0.3462220 & -1.0795080 & -0.5533110 \\ \mathrm{O} & 2.5187390 & -0.1928580 & -0.5708820 \\ \mathrm{H} & 0.7888180 & 1.6284110 & -0.0587570 \\ \mathrm{C} & 0.6885140 & 0.8780520 & 0.7378510 \\ \mathrm{C} & 1.3342930 & -0.4317190 & 0.2556260 \\ \mathrm{H} & 1.6886600 & -1.0450360 & 1.1067490 \\ \mathrm{H} & 1.1352080 & 1.2861440 & 1.6533430 \\ \mathrm{O} & -1.5202160 & -2.0181520 & 1.1581220 \\ \mathrm{H} & -1.4334430 & -2.8294880 & 0.6287350 \\ \mathrm{C} & 3.6746840 & 0.0508720 & 0.0926270 \\ \mathrm{H} & 3.6629610 & -0.1959260 & 1.1558320 \\ \mathrm{C} & 4.7748480 & 0.5332250 & -0.5042510 \\ \mathrm{H} & 4.7911440 & 0.7875230 & -1.5608000 \\ \mathrm{H} & 5.6834760 & 0.6681770 & 0.0726570\end{array}$

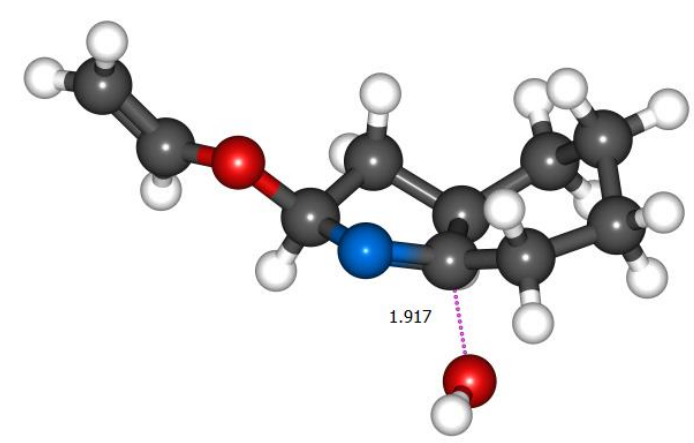

Transition state of nucleophilic addition of hydroxide anion to the $\mathrm{C}=\mathrm{N}$ bond of 5-vinyloxypyrroline $\left(\mathrm{TS}_{19 \rightarrow 20}\right)$ $I 268 \mathrm{~cm}^{-1}$

a.u.

$E$ (B2PLYP) $-595.4955597$ $-595.2488037$ $-595.2350097$ $E(\mathrm{~B} 2 \mathrm{PLYP})+H_{\text {corr }}$ $E(\mathrm{~B} 2 \mathrm{PLYP})+G_{c o r r}$ $-595.2885497$
$-2.1258180$
$-1.9651600$
$-2.1316570$
$-1.1331020$
$-1.3512610$
$-1.3440860$
$-2.6827090$
$-0.9627000$
$-1.9811720$
$-3.1568930$
$-1.2390690$
$-0.1082260$
$-0.5996010$
$-2.3255720$
$-1.8215630$
$-1.5359150$
4.4346760
0.7373730
0.0218820
$-0.3889010$
0.2427630
0.5115530
$-3.5248510$
$-3.6587650$
5.1088840
5.1106230
5.8335130
5.9096340
6.3513630
$-0.2383120$
$-1.3272120$
$-0.7920030$
0.3417670
1. 5019580
1.0718380
$-2.1273200$
$-1.7618030$
$-1.6138560$
$-0.4259460$
0.7104270
$-0.0477110$
2. 2874850
1. 9610460
1.8681050
$-0.7362480$
$-1.1141780$
0.3198510
0.7943100
$-0.1866300$
$-0.4399710$
1.6871100
$-0.0214800$
0.6729170
$-0.0216990$
0.6801120
0.4057060
$-0.1923230$
1. 3625300

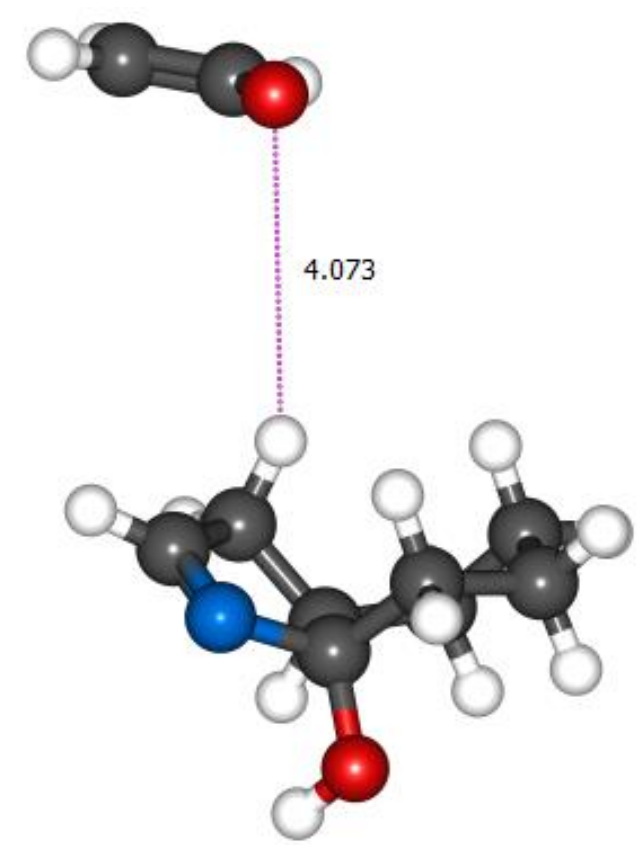

Complex of azolin-2-ol and vinyl alcohol anion 20

a.u.

$E$ (B2PLYP)

$E(\mathrm{~B} 2 \mathrm{PLYP})+Z P E$

$-595.5295234$

$-595.2832894$

$-595.2679654$

$-595.3309384$ 


$\begin{array}{llll}\mathrm{C} & 0.4759560 & -0.7036090 & 0.1780890 \\ \mathrm{C} & -0.5201260 & -1.0990570 & -0.9269920 \\ \mathrm{C} & -1.9694380 & -0.7144530 & -0.5978730 \\ \mathrm{C} & -2.0770610 & 0.7930450 & -0.3301160 \\ \mathrm{C} & -1.1857450 & 1.1994150 & 0.8531790 \\ \mathrm{C} & 0.2768270 & 0.7475390 & 0.7095950 \\ \mathrm{H} & -0.4185650 & -2.1760360 & -1.1057790 \\ \mathrm{H} & -0.2220120 & -0.5920500 & -1.8533390 \\ \mathrm{H} & -2.6176350 & -1.0033700 & -1.4348970 \\ \mathrm{H} & -2.3177420 & -1.2728730 & 0.2818580 \\ \mathrm{H} & -3.1166070 & 1.0724830 & -0.1164340 \\ \mathrm{H} & -1.7855030 & 1.3442250 & -1.2363500 \\ \mathrm{H} & -1.2172860 & 2.2861120 & 1.0073890 \\ \mathrm{H} & -1.5969530 & 0.7468700 & 1.7663220 \\ \mathrm{H} & 0.7449140 & 0.7808230 & 1.7046420 \\ \mathrm{~N} & 1.8489870 & -0.7143740 & -0.3952550 \\ \mathrm{H} & 0.6607660 & 1.9187930 & -1.1271120 \\ \mathrm{C} & 1.1917640 & 1.5583950 & -0.2331090 \\ \mathrm{C} & 2.1941330 & 0.4975110 & -0.6219900 \\ \mathrm{H} & 3.1569080 & 0.7239580 & -1.0817910 \\ \mathrm{H} & 1.6566370 & 2.4319860 & 0.2373520 \\ \mathrm{O} & 0.3561340 & -1.6850950 & 1.2019400 \\ \mathrm{H} & 0.9632270 & -1.4522590 & 1.9247110\end{array}$

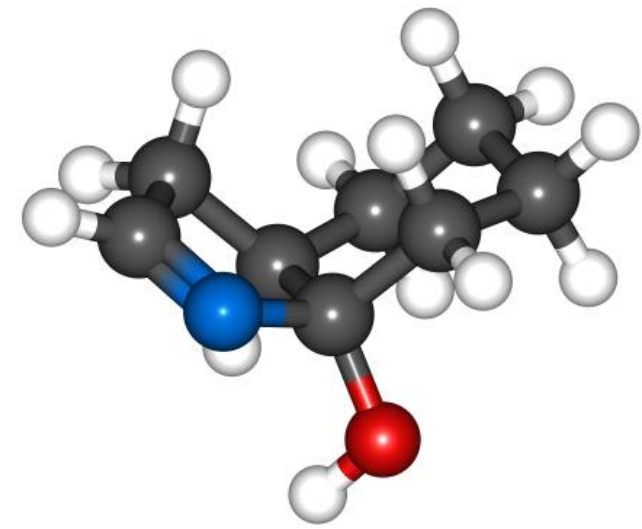

Azolin-2-ol 21

$E$ (B2PLYP)

$E(\mathrm{~B} 2 \mathrm{PLYP})+Z P E$

$E(\mathrm{~B} 2 \mathrm{PLYP})+H_{\text {corr }}$

$E(\mathrm{~B} 2 \mathrm{PLYP})+G_{\text {corr }}$ a.u. $-442.3703193$ $-442.1671023$ $-442.1575553$ $-442.1999233$

$\begin{array}{lll}-1.0308160 & -0.6460580 & -0.2173490 \\ -0.8501370 & 0.3788350 & -1.3508350 \\ -1.0436610 & 1.8293100 & -0.8866250 \\ -0.0730970 & 2.1620910 & 0.2549430 \\ -0.3006590 & 1.2277840 & 1.4526920 \\ -0.2746640 & -0.2667860 & 1.0903640 \\ -1.5431510 & 0.1178610 & -2.1596640 \\ 0.1655650 & 0.2684500 & -1.7505520 \\ -0.8813940 & 2.5042780 & -1.7367710 \\ -2.0788460 & 1.9834630 & -0.5515620 \\ -0.2015270 & 3.2040100 & 0.5758910 \\ 0.9600780 & 2.0709120 & -0.1088530 \\ 0.4378790 & 1.4232860 & 2.2414810 \\ -1.2836940 & 1.4581950 & 1.8876040 \\ -0.7689750 & -0.8200660 & 1.9033830 \\ -0.4319450 & -1.9394360 & -0.6430010 \\ 1.8419170 & -0.2902970 & 0.3811700 \\ 1.0982600 & -0.9399280 & 0.8712350 \\ 0.7038360 & -2.0576290 & -0.0616340 \\ 1.3394460 & -2.9212980 & -0.2623160 \\ 1.5597480 & -1.3090340 & 1.7950330 \\ -2.4352700 & -0.7888580 & -0.0212030 \\ -2.5808470 & -1.4226030 & 0.7015260 \\ 3.9320330 & 0.3421330 & -0.5277350 \\ 4.7089440 & 0.7569850 & -0.9406030\end{array}$

$-1.0308160$
$-0.6460580$ 0.3788350 1.8293100 2.1620910 0.2684500 2.5042780 .9834630 3.2040100 $-0.8200660$ $-1.9394360$ 1.3090340 7888580 0.3421330 0.7569850
$-0.2173490$ .2549430 (526920 $-2.1596640$ $-1.7505520$ 0.5758910 2. 2414810 8876040 0.3811700 $-0.0616340$ $-0.2623160$ $-0.0212030$ $-0.9406030$

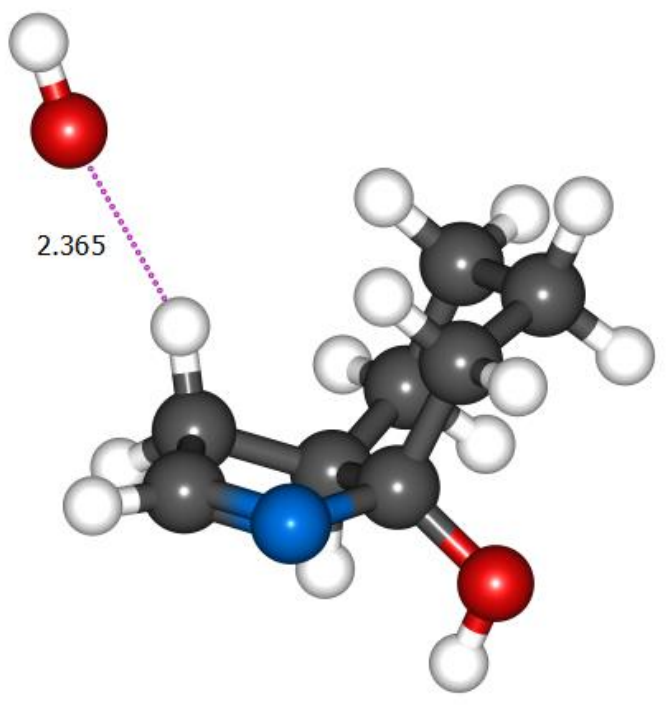

Complex of azolin-2-ol and hydroxide ion 22

a.u.

$E$ (B2PLYP)

$E(\mathrm{~B} 2 \mathrm{PLYP})+Z P E$

$E(\mathrm{~B} 2 \mathrm{PLYP})+H_{\text {corr }}$ $E(\mathrm{~B} 2 \mathrm{PLYP})+G_{\text {corr }}$
$-518.1628008$ $-517.9492968$ $-517.9364418$ $-517.9875638$ 
$-0.8861650$

$-0.9879840$

$-1.2633000$

$-0.1752490$

$-0.1373060$

$-0.0041820$

$-1.7592440$

$-0.0338650$

$-1.2985490$

$-2.2495200$

$-0.3572020$

0.7987170

0.6810530

$-1.0687610$

$-0.3619600$

$-0.2312930$

2. 2262130

1. 3756140

0.9879000

1. 6732080

2.0441190

$-2.2418140$

$-2.2009090$

3.0164950

3.8723370
$-0.7529130$

0.2708980

1. 7047470

2.1564220

1.2306230

$-0.2664360$

$-0.0775590$

0.2573520

2. 3760700

1. 7613840

3.1904890

2.1435120

1. 5209740

1.3764320

$-0.8271420$

$-1.9812660$

0.0473980

$-0.8327890$

$-1.9590090$

$-2.7664140$

$-1.0827420$

$-1.0262980$

$-1.7211380$

0.7805800

0.3267460
$-0.1298620$

$-1.2757660$

$-0.8029970$

0.1808610

1. 4051990

1.0635830

$-1.9748550$

$-1.8163960$

$-1.6721250$

$-0.3198130$

0.5056820

$-0.3249630$

2.0791460

1. 9739530

1.9490100

$-0.6255890$

$-0.0875090$

0.6708540

$-0.1482630$

$-0.4233880$

1. 5065290

0.2732460

0.9520500

$-0.6691800$

$-0.5823830$

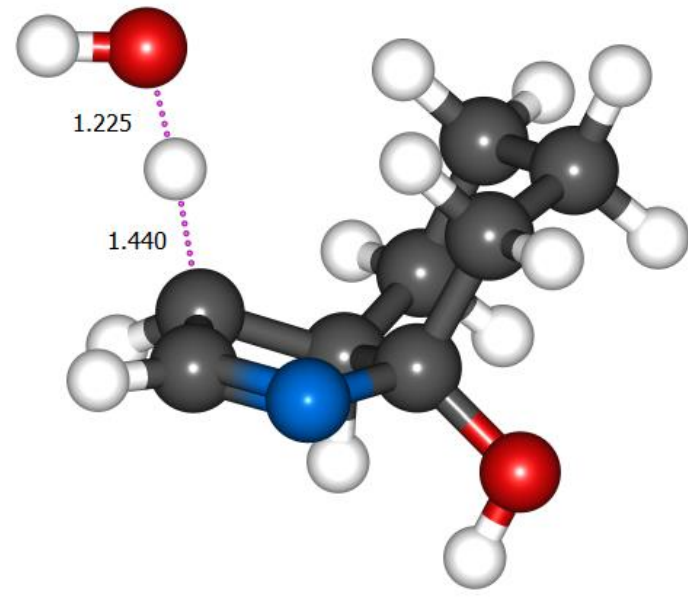

Transition state of proton detachment by hydroxide ion $\left(\mathrm{TS}_{22 \rightarrow 23}\right)$

$I 1353 \mathrm{~cm}^{-1}$

a.u.

$E$ (B2PLYP)

$E(\mathrm{~B} 2 \mathrm{PLYP})+Z P E$

$E(\mathrm{~B} 2 \mathrm{PLYP})+H_{\text {corr }}$

$E(\mathrm{~B} 2 \mathrm{PLYP})+G_{\text {corr }}$
$-518.17482$

$-517.954803$

$-518.003484$
$-517.966901$
$-0.9281090$

$-0.8568220$

$-1.1055640$

$-0.1059170$

$-0.2465730$

$-0.1511550$

$-1.5642790$

0.1499850

$-1.0157220$

$-2.1317690$

$-0.2653350$

0.9141280

0.5048040

$-1.2270970$

$-0.6310190$

$-0.2947860$

2.5069330

1. 2128160

0.9026080

1. 5920490

1.9324270

$-2.3375100$

$-2.3948140$

3. 2564390

4.0140750
$-0.7195370$

0.3333780

1. 7755430

2.1568390

1. 2117440

$-0.2858870$

0.0365180

0.2752150

2. 4601920

1.8789080

3.1950590

2.1024750

1. 4508800

1.3981080

$-0.8344440$

$-1.9678120$

0.1255660

$-0.9198100$

$-1.9779770$

$-2.7943040$

$-1.0660830$

$-0.9152860$

$-1.6890720$

0.6354120

0.0288990
$-0.1880310$

$-1.3132450$

$-0.8494440$

0.2509890

1. 4522370

1.0989450

$-2.0984460$

$-1.7455020$

$-1.7046030$

$-0.4678020$

0.5745530

$-0.1533230$

2. 2186930

1. 9184350

1.9389550

$-0.6374780$

$-0.1993190$

0.8270980

$-0.0446690$

$-0.2813620$

1.6368700

0.0742100

0.6606230

$-0.6510520$

$-0.6239600$

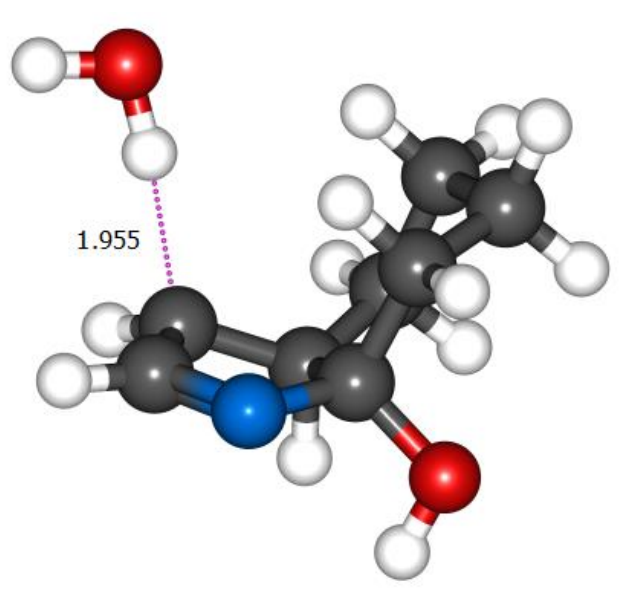

Complex of azolin-2-ol carbanion and water molecule 23

$E$ (B2PLYP)

a.u.

$E(\mathrm{~B} 2 \mathrm{PLYP})+Z P E$

$E$ (B2PLYP $)+H_{\text {corr }}$

$E(\mathrm{~B} 2 \mathrm{PLYP})+G_{\text {corr }}$
$-518.1855364$

$-517.9740314$

$-517.9612354$

$-518.0118124$ 


$\begin{array}{ll}\mathrm{C} & 0.5278260 \\ \mathrm{C} & -0.5021330 \\ \mathrm{C} & -1.9593570 \\ \mathrm{C} & -2.0874710 \\ \mathrm{C} & -1.1939430 \\ \mathrm{C} & 0.2836670 \\ \mathrm{H} & -0.3820980 \\ \mathrm{H} & -0.2229900 \\ \mathrm{H} & -2.6129810 \\ \mathrm{H} & -2.2957060 \\ \mathrm{H} & -3.1315790 \\ \mathrm{H} & -1.7936110 \\ \mathrm{H} & -1.2491620 \\ \mathrm{H} & -1.6019280 \\ \mathrm{H} & 0.7070930 \\ \mathrm{~N} & 1.8759180 \\ \mathrm{C} & 1.2074460 \\ \mathrm{C} & 2.1356080 \\ \mathrm{H} & 3.0585200 \\ \mathrm{H} & 1.4009210 \\ \mathrm{O} & 0.4131830 \\ \mathrm{H} & 1.2167910\end{array}$

$-0.6696150$

$-1.0947530$

$-0.7366490$

0.7741530

1.2065930

0.7871720

$-2.1722450$

$-0.5840900$

$-1.0536620$

$-1.2798990$

1.0487710

1.3071870

2.2956880

0.7576840

0.8098810

$-0.6741580$

1.5643510

0.6310230

0.8817230

2.6324570

$-1.6459650$

$-1.5303200$
0.1634050

$-0.9070690$

$-0.5819740$

$-0.3454770$

0.8247890

0.6883040

$-1.0799100$

$-1.8379400$

$-1.4072330$

0.3133950

$-0.1396290$

$-1.2615320$

0.9680610

1. 7445840

1.7183930

$-0.4140300$

$-0.2357600$

$-0.6681230$

$-1.1997520$

$-0.1500420$

1. 2294540

1. 7656220

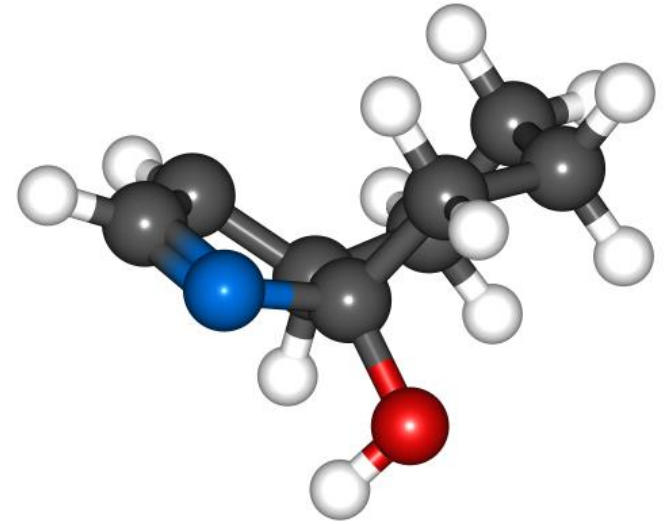

Azolin-2-ol carbanion 24

a.u.

E(B2PLYP)

$E$ (B2PLYP) $+Z P E$

$E$ (B2PLYP $)+H_{\text {corr }}$

$E(\mathrm{~B} 2 \mathrm{PLYP})+G_{\text {corr }}$
$-441.7675006$

$-441.5794436$

$-441.5698876$

$-441.6121656$
$-2.1098390$

$-1.9020890$

$-2.0506630$

$-1.0725940$

$-1.3357790$

$-1.3495110$

$-2.6056490$

$-0.8922820$

$-1.8668420$

$-3.0806450$

$-1.1669370$

$-0.0410970$

$-0.5984480$

$-2.3151530$

$-1.8558180$

$-1.5368170$

5.4270040

0.7473740

0.0078110

$-0.4048530$

0.2139160

0.4706220

$-3.5160630$

$-3.6797770$

5.3026640

5.8880160

4.5443940

3. 9280050

4.5476600
$-0.6254080$

0.4178530

1.8640280

2.1444320

1.1965310

$-0.2904600$

0.1942210

0.2860190

2.5500470

2.0438670

3. 1837870

2.0276670

1. 3557880

1.4466150

$-0.8494750$

$-1.9224390$

$-1.0569970$

$-0.3277110$

$-0.9882080$

$-2.0781170$

$-2.9496830$

$-1.3783190$

$-0.7410580$

$-1.3843440$

0.2060630

0.5982040

1.1483780

0.8900500

2.1842220
$-0.2436140$

$-1.3563330$

$-0.8627370$

0.2863310

1. 4660180

1.0745510

$-2.1669950$

$-1.7647220$

$-1.6993670$

$-0.5247650$

0.6260060

$-0.0770860$

2.2638780

1.8974390

1.8754640

$-0.6947220$

$-0.1254100$

0.3663840

0.8435470

$-0.1169800$

$-0.3336650$

1.7568020

$-0.0544400$

0.6559510

0.1201630

0.9845620

$-0.5369630$

$-1.3989010$

$-0.2030280$

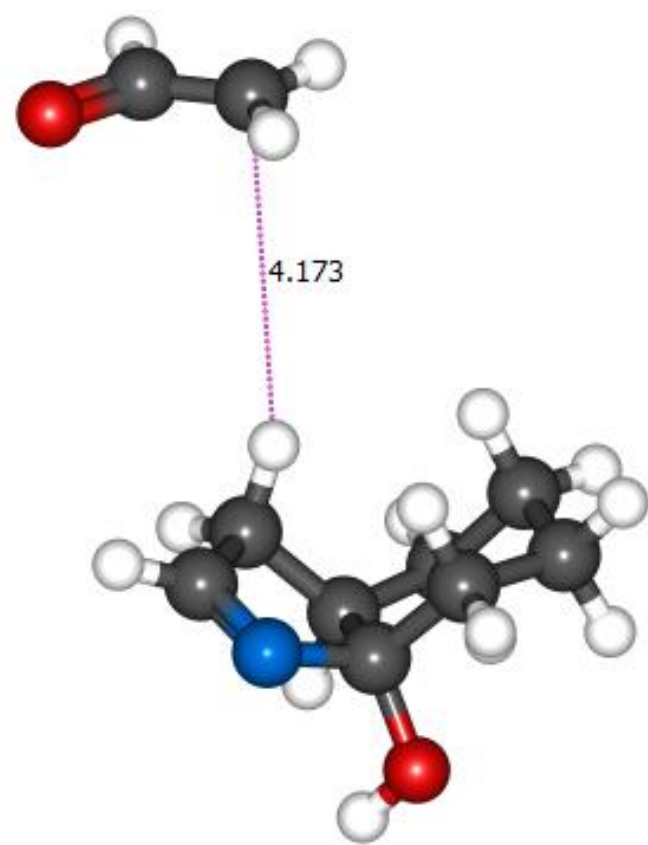

Complex of azolin-2-ol and vinyl alcohol anion 25

$E$ (B2PLYP)

$E(\mathrm{~B} 2 \mathrm{PLYP})+Z P E$

$E(\mathrm{~B} 2 \mathrm{PLYP})+H_{\text {corr }}$

$E(\mathrm{~B} 2 \mathrm{PLYP})+G_{\text {corr }}$ a.u.

$-595.5296979$

$-595.2835199$

$-595.2681319$

$-595.3321949$ 


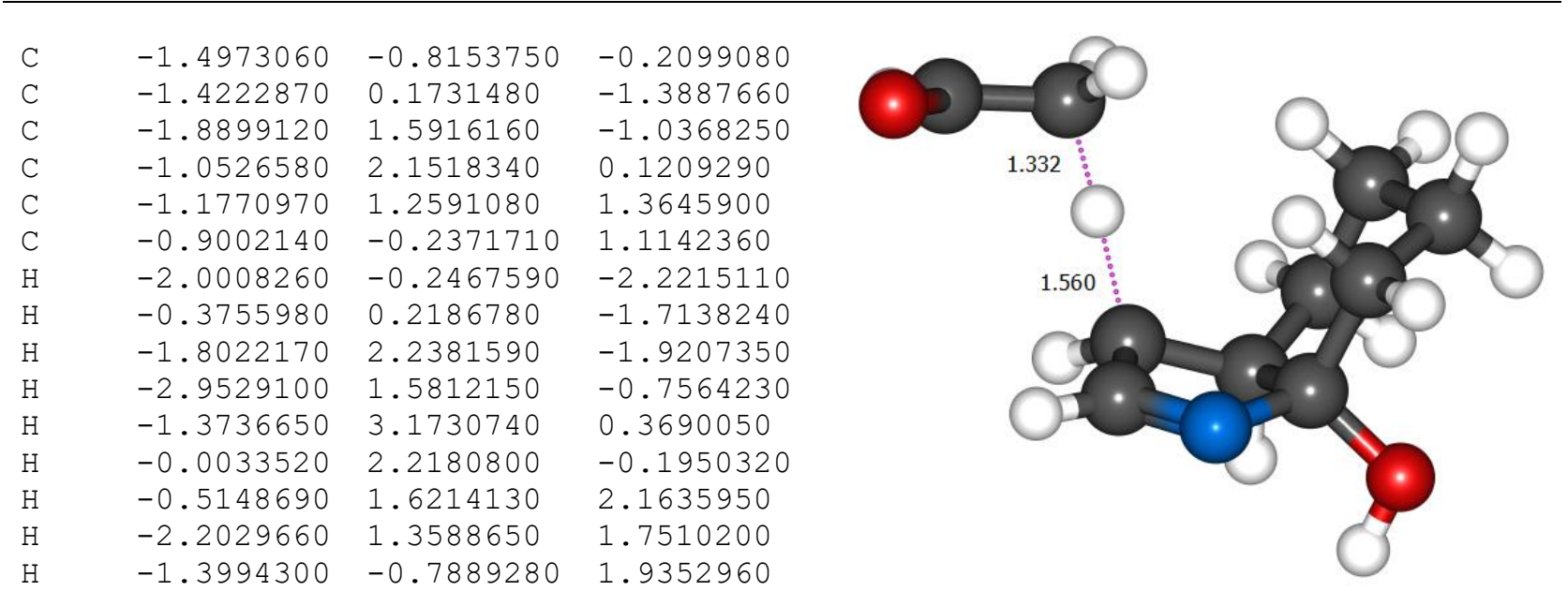

$\begin{array}{lll}-1.3994300 & -0.7889280 & 1.9352960\end{array}$

$-0.6944630-2.0138540-0.5151940$

$4.3363950-0.4642920-0.6837820$

$\begin{array}{lll}1.5986600 & 0.2077090 & 0.3818570\end{array}$

$\begin{array}{llll}0.5461610 & -0.7436500 & 1.0294320\end{array}$

$\begin{array}{llll}0.4065840 & -1.9023970 & 0.2008930\end{array}$

$\begin{array}{lll}1.1776430 & -2.6711790 & 0.1014470\end{array}$

$\begin{array}{lll}1.1077910 & -0.8562550 & 1.9637360\end{array}$

$-2.8914820-1.1557410-0.0728410$

$\begin{array}{llll}-2.9425510 & -1.8632260 & 0.5921760\end{array}$

$\begin{array}{lll}3.8016830 & 0.3804380 & 0.0735610\end{array}$

$4.3021550 \quad 0.5921310 \quad 1.0438410$

$2.5310360 \quad 1.0155750 \quad-0.1205120$

$2.2072780 \quad 1.0915610-1.1633380$

$\begin{array}{lll}2.4008550 & 1.9439500 & 0.4424570\end{array}$

Transition state of proton detachment by vinyl alcohol anion $\left(\mathrm{TS}_{25 \rightarrow 26}\right)$ $I 1264 \mathrm{~cm}^{-1}$

$E$ (B2PLYP)

$E(\mathrm{~B} 2 \mathrm{PLYP})+Z P E$

$E(\mathrm{~B} 2 \mathrm{PLYP})+H_{\text {corr }}$

$E(\mathrm{~B} 2 \mathrm{PLYP})+G_{\text {corr }}$

a.u.

$-595.5142482$

$-595.2727062$

$-595.2587252$

$-595.3128802$
$-1.9500140$
$-1.5958280$
$-2.0615880$
$-1.4749190$
$-1.9305130$
$-1.6525910$
$-1.9985220$
$-0.5017280$
$-1.7521890$
$-3.1599120$
$-1.7785640$
$-0.3773000$
$-1.4670190$
$-3.0151960$
$-2.3512830$
$-1.1305520$
6.0325650
3.0986110
$-0.2496600$
$-0.1587650$
0.6454920
0.3013810
$-3.3586340$
$-3.5186990$
5.1760380
5.1966380
4.0612790
4.1072690
4.1127960
$-0.7473370$
0.3567970
1. 7664750
2.1541520
1. 1613230
$-0.3188710$
0.0550990
0.3675460
2. 4871140
1. 8052580
3.1729620
2. 1579430
1. 4059200
1.2909610
$-0.9135630$
$-1.9373860$
$-0.4557670$
0.4860670
$-0.8801400$
$-1.8810210$
$-2.6217600$
$-0.8747630$
$-1.0282000$
$-1.8685200$
0.0672540
$-0.0853820$
0.9254230
1.0319690
1. 9122660
$-0.3795240$
$-1.3991390$
$-1.0097190$
0.3544900
1. 4326810
1.0979700
$-2.3748800$
$-1.4878600$
$-1.7802110$
$-0.9636550$
0.6333830
0.2853130
2.3996250
1.5747700
1.7295340
$-0.6318430$
$-0.4425870$
0.0340720
1. 2644050
0.3098320
0.2693730
2.2038310
$-0.5792020$
$-0.1155360$
0.2531130
1. 3508470
$-0.2623330$
$-1.3490790$
0.2170290

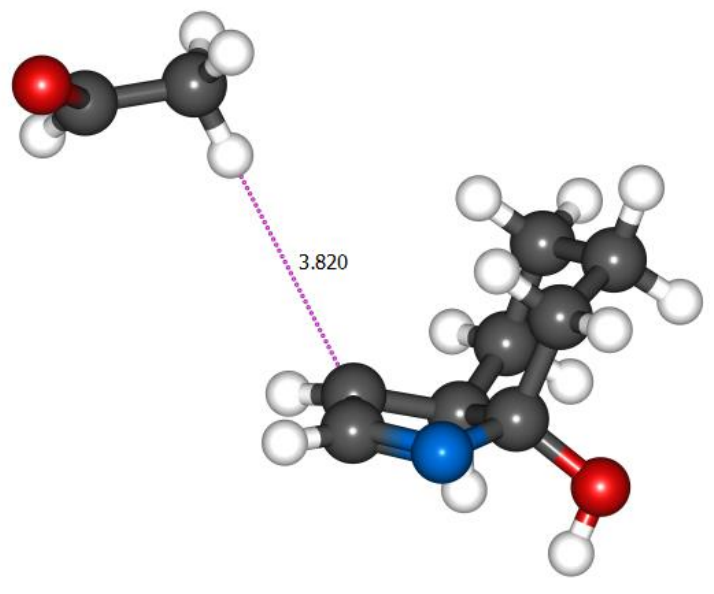

Complex of azolin-2-ol carbanion and acetaldehyde molecule 26

a.u.

$E$ (B2PLYP)

$-595.5208824$

$E(\mathrm{~B} 2 \mathrm{PLYP})+Z P E$

$E(\mathrm{~B} 2 \mathrm{PLYP})+H_{\text {corr }}$

$E(\mathrm{~B} 2 \mathrm{PLYP})+G_{\text {corr }}$

$-595.2768154$

$-595.2608294$

$-595.3252214$ 


$\begin{array}{lll}-0.5850500 & -0.6343890 & -0.2038440 \\ 0.6217720 & -1.5093140 & 0.1451180 \\ 1.6331810 & -0.7124690 & 0.9734100 \\ 2.3061090 & 0.3535240 & 0.0982480 \\ 1.3178170 & 1.2022460 & -0.7585370 \\ -0.1994300 & 0.7991400 & -0.7581130 \\ 1.1045430 & -1.8726720 & -0.7750960 \\ 0.2566740 & -2.3885740 & 0.6926730 \\ 1.1071050 & -0.2473970 & 1.8185290 \\ 2.3995380 & -1.3742190 & 1.4001590 \\ 3.0033580 & -0.1640750 & -0.5759620 \\ 2.9244470 & 1.0127220 & 0.7219750 \\ 1.3731910 & 2.2501920 & -0.4379020 \\ 1.6787190 & 1.1939000 & -1.7960490 \\ -0.5142770 & 0.8069180 & -1.8131740 \\ -1.4713530 & -0.4632130 & 0.9372880 \\ -1.0926340 & 1.6812630 & 0.0913210 \\ -1.7477020 & 0.8703790 & 0.9847270 \\ -2.4555370 & 1.2264370 & 1.7384400 \\ -1.1966050 & 2.7575240 & -0.0178880 \\ -1.3406630 & -1.3389970 & -1.2664940 \\ -2.1807540 & -1.5485730 & -0.8187410\end{array}$

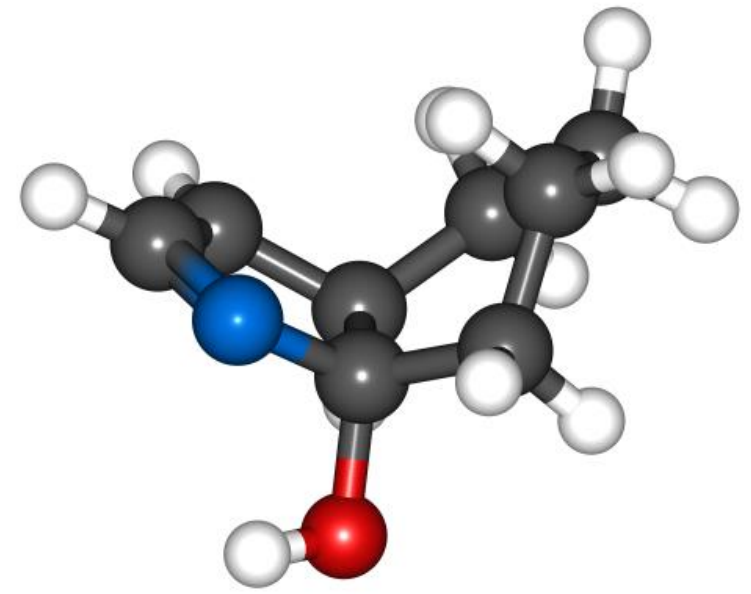

Transition state of conformation change of azolin-2-ol carbanion $\left(\mathrm{TS}_{24 \rightarrow 27}\right)$ $I 148 \mathrm{~cm}^{-1}$

.u.

$E$ (B2PLYP)

$E(\mathrm{~B} 2 \mathrm{PLYP})+Z P E$ $-441.7627072$

$E(\mathrm{~B} 2 \mathrm{PLYP})+H_{\text {corr }}$ $E(\mathrm{~B} 2 \mathrm{PLYP})+G_{\text {corr }}$ $-441.5753632$ $-441.5661632$ $-441.6079472$

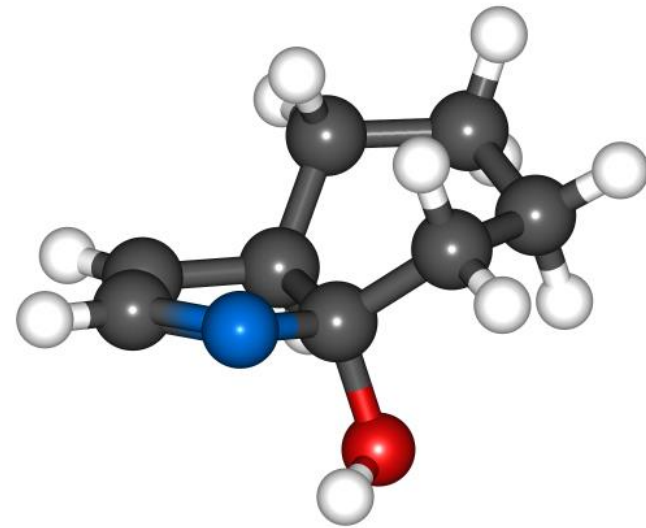

\section{Azolin-2-ol carbanion 27}

a.u.

$E$ (B2PLYP)

$E(\mathrm{~B} 2 \mathrm{PLYP})+Z P E$

$E(\mathrm{~B} 2 \mathrm{PLYP})+H_{\text {corr }}$

$E(\mathrm{~B} 2 \mathrm{PLYP})+G_{\text {corr }}$
$-441.7675816$

$-441.5801246$

$-441.5701966$

$-441.6135706$ 


$\begin{array}{lll}-0.3921090 & 0.5205270 & -0.2936670 \\ 0.8880530 & 1.0779280 & -0.8784670 \\ 2.0975780 & 0.6480210 & -0.0379800 \\ 2.2193480 & -0.8946340 & 0.0702650 \\ 0.8480640 & -1.6177220 & 0.0269200 \\ -0.2855760 & -0.7267000 & 0.5931390 \\ 0.8293570 & 2.1674080 & -0.9587290 \\ 0.9929920 & 0.6863270 & -1.9035720 \\ 3.0222450 & 1.0658690 & -0.4565730 \\ 1.9786200 & 1.0794770 & 0.9624060 \\ 2.7418720 & -1.1449390 & 1.0032180 \\ 2.8476600 & -1.2783070 & -0.7448060 \\ 0.5944780 & -1.8796930 & -1.0094560 \\ 0.8966930 & -2.5629240 & 0.5822440 \\ -0.0355310 & -0.4543990 & 1.6241460 \\ -1.5384250 & 0.5388250 & -0.9820600 \\ -1.6843180 & -1.2783240 & 0.4617060 \\ -2.3238450 & -0.4976410 & -0.4443550 \\ -3.3471530 & -0.6138670 & -0.7969400 \\ -2.0834270 & -2.1356780 & 0.9933080 \\ -0.5740990 & 1.9357820 & 1.2279470 \\ -1.2792050 & 2.4239660 & 0.7702340\end{array}$

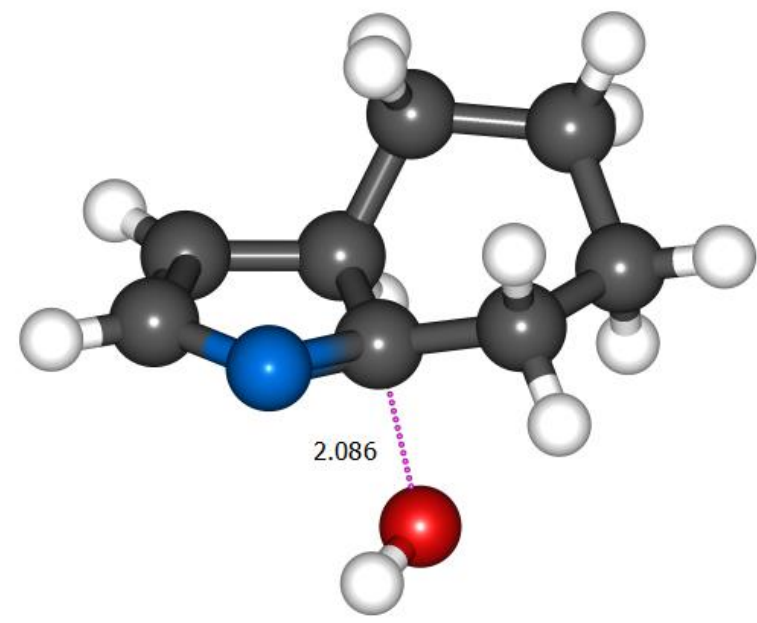

Transition state of hydroxide ion elimination

$\left(\mathrm{TS}_{27 \rightarrow 28}\right)$

I254 $\mathrm{cm}^{-1}$

$E$ (B2PLYP)

a.u.

$E(\mathrm{~B} 2 \mathrm{PLYP})+Z P E$

$-441.7449552$

$E(\mathrm{~B} 2 \mathrm{PLYP})+H_{\text {corr }}$

$E(\mathrm{~B} 2 \mathrm{PLYP})+G_{\text {corr }}$

$-441.5594802$

$-441.5493452$

$-441.5932082$

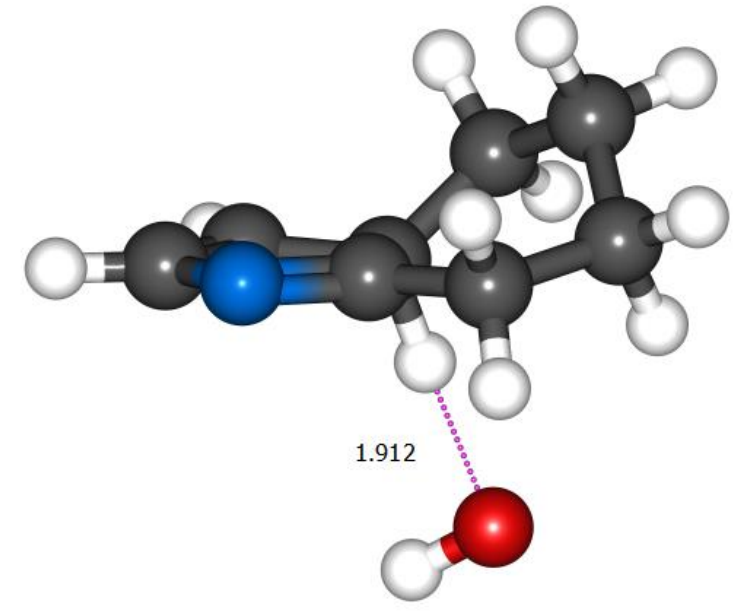

Complex of $3 \mathrm{H}$-pyrrole and hydroxide ion 28

a.u.

$E(\mathrm{~B} 2 \mathrm{PLYP})$

$E(\mathrm{~B} 2 \mathrm{PLYP})+Z P E$

$E(\mathrm{~B} 2 \mathrm{PLYP})+H_{\text {corr }}$

$E(\mathrm{~B} 2 \mathrm{PLYP})+G_{\text {corr }}$
$-441.7503776$

$-441.5659056$

$-441.5543126$

$-441.6024506$ 


\begin{tabular}{llll}
\hline & & & \\
$\mathrm{C}$ & 0.2494170 & -0.8147320 & 0.4209200 \\
$\mathrm{C}$ & -1.0547310 & -1.1220380 & 1.1031270 \\
$\mathrm{C}$ & -2.1909930 & -0.2352110 & 0.5498970 \\
$\mathrm{C}$ & -2.0656660 & -0.0674400 & -0.9734020 \\
$\mathrm{C}$ & -0.7999170 & 0.7269150 & -1.3645580 \\
$\mathrm{C}$ & 0.4057710 & 0.3456670 & -0.4743560 \\
$\mathrm{H}$ & -0.9631130 & -0.9955280 & 2.1918160 \\
$\mathrm{H}$ & -1.2950270 & -2.1825150 & 0.9396770 \\
$\mathrm{H}$ & -3.1605590 & -0.6776450 & 0.8099570 \\
$\mathrm{H}$ & -2.1586460 & 0.7545590 & 1.0261690 \\
$\mathrm{H}$ & -2.9557540 & 0.4327930 & -1.3765720 \\
$\mathrm{H}$ & -2.0297490 & -1.0659210 & -1.4350480 \\
$\mathrm{H}$ & -0.5659480 & 0.5441120 & -2.4209880 \\
$\mathrm{H}$ & -1.0047970 & 1.8018350 & -1.2724260 \\
$\mathrm{H}$ & 0.5272280 & 1.2593720 & 0.3116990 \\
$\mathrm{~N}$ & 1.3573730 & -1.5101820 & 0.5643140 \\
$\mathrm{C}$ & 1.7816990 & 0.1432810 & -0.9870250 \\
$\mathrm{C}$ & 2.3001370 & -0.9133350 & -0.3016380 \\
$\mathrm{H}$ & 3.3079870 & -1.3152440 & -0.3454040 \\
$\mathrm{H}$ & 2.2999260 & 0.7843650 & -1.6926420 \\
$\mathrm{O}$ & 0.6453740 & 2.5447040 & 1.2455970 \\
$\mathrm{H}$ & 1.5795410 & 2.4948070 & 1.5109950 \\
& & &
\end{tabular}

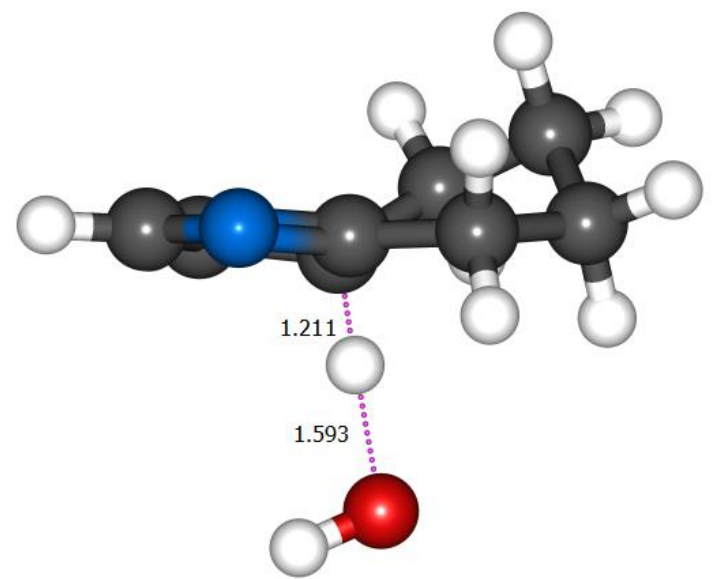

Transition state of proton detachment

$\left(\mathrm{TS}_{28 \rightarrow 29}\right)$

$I 190 \mathrm{~cm}^{-1}$
$E$ (B2PLYP)
$E(\mathrm{~B} 2 \mathrm{PLYP})+Z P E$
$E(\mathrm{~B} 2 \mathrm{PLYP})+H_{\text {corr }}$
$E(\mathrm{~B} 2 \mathrm{PLYP})+G_{\text {corr }}$

a.u.

$-441.7589919$

$-441.5765599$

$-441.5660099$

$-441.6117279$

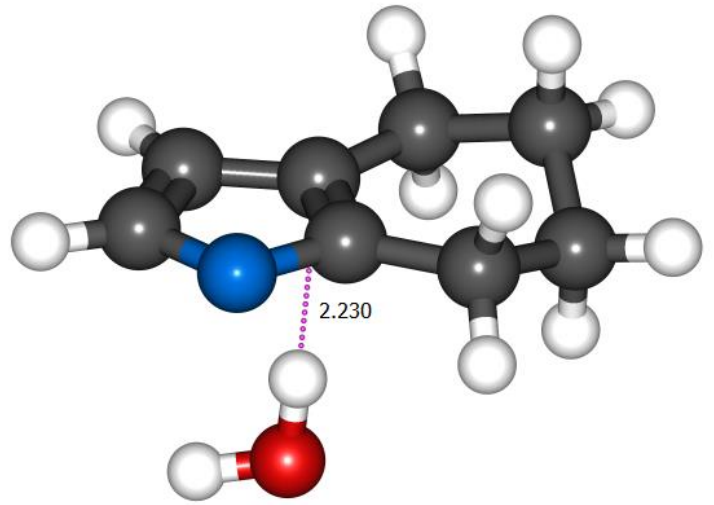

Complex of $1 H$-pyrrole ion and water molecule 29

a.u.

$E$ (B2PLYP)

$-441.8141121$

$E(\mathrm{~B} 2 \mathrm{PLYP})+Z P E$

$E(\mathrm{~B} 2 \mathrm{PLYP})+H_{\text {corr }}$

$-441.6290281$

$-441.6171831$

$-441.6665211$ 


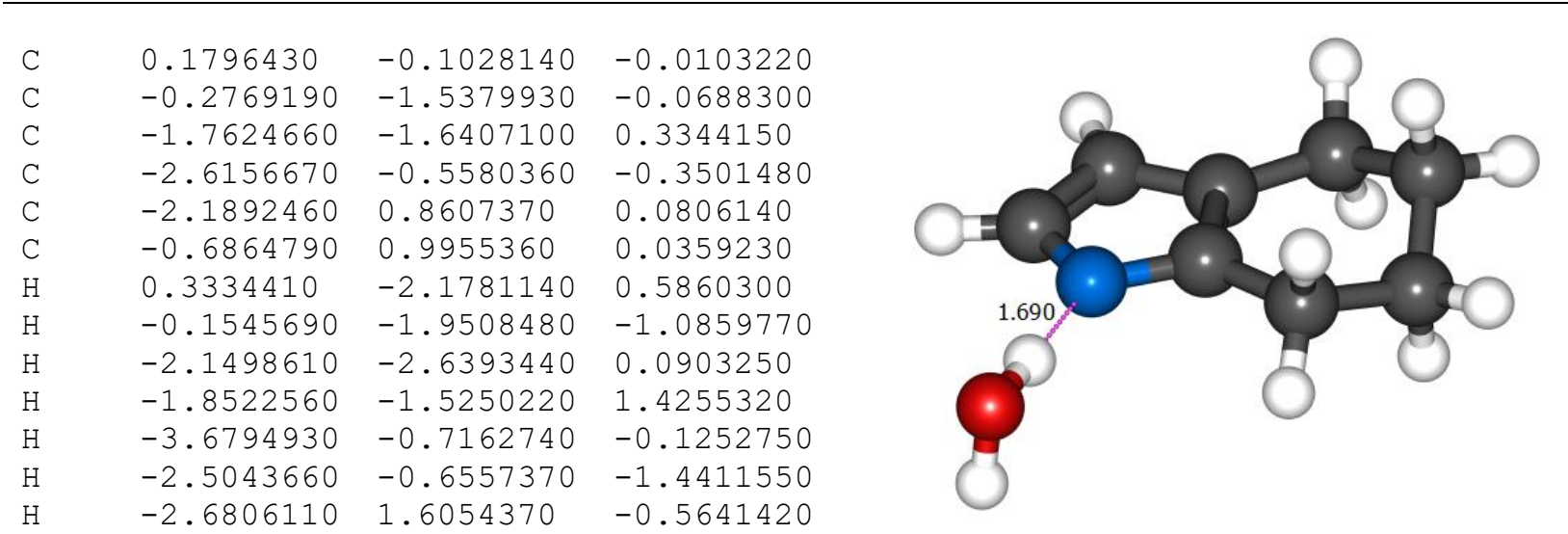

$\begin{array}{lll}-2.6806110 & 1.6054370 & -0.5641420\end{array}$

2.8978670

1.4928490

0.1504290

$-0.6409070$

0.2960840

2.1499880

1.4659250

1.6679640

2.3916630

$-0.1623850$

2.2383860

3. 1905770

3.7587360

$-1.1912960$

4.0254200

$-1.3289060$

$-0.0820270$

$-0.0310360$

0.0488050

0.0070480

0.0017890

0.0851970

$-0.1286270$

0.7934930

\section{Complex of $1 \mathrm{H}$-pyrrole ion and water molecule 30}

$E$ (B2PLYP)

$E(\mathrm{~B} 2 \mathrm{PLYP})+Z P E$

$E(\mathrm{~B} 2 \mathrm{PLYP})+H_{\text {corr }}$

$E(\mathrm{~B} 2 \mathrm{PLYP})+G_{\text {corr }}$ a.u. $-441.8233663$ $-441.6379903$ $-441.6267363$ $-441.6748413$

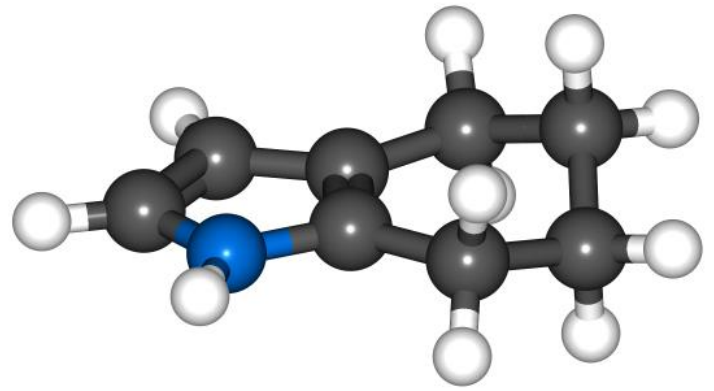

4,5,6,7-tetrahydroindole 31

a.u.

E(B2PLYP)

$-365.980306$

$-365.804177$

$-365.795889$

$E$ (B2PLYP $)+H_{\text {corr }}$

$E(\mathrm{~B} 2 \mathrm{PLYP})+G_{\text {corr }}$

$-365.835704$ 


$\begin{array}{llll}\mathrm{C} & -0.2662130 & -0.5722460 & 0.0464110 \\ \mathrm{C} & -0.7695300 & -0.8595170 & -1.3445790 \\ \mathrm{C} & -2.3000970 & -0.6751360 & -1.4031880 \\ \mathrm{C} & -2.7456290 & 0.6216660 & -0.7038550 \\ \mathrm{C} & -2.4087680 & 0.6043050 & 0.8017570 \\ \mathrm{C} & -0.9996200 & 0.1108640 & 1.0223690 \\ \mathrm{H} & -0.5026660 & -1.8790350 & -1.6607340 \\ \mathrm{H} & -0.3003320 & -0.1856500 & -2.0828830 \\ \mathrm{H} & -2.6390550 & -0.6777750 & -2.4480820 \\ \mathrm{H} & -2.7884490 & -1.5296550 & -0.9100120 \\ \mathrm{H} & -3.8240170 & 0.7764650 & -0.8458360 \\ \mathrm{H} & -2.2378140 & 1.4741880 & -1.1803550 \\ \mathrm{H} & -2.5542760 & 1.6101890 & 1.2241360 \\ \mathrm{H} & -3.1391260 & -0.0417970 & 1.3195120 \\ \mathrm{H} & 2.2313340 & -1.7625020 & -0.2801410 \\ \mathrm{~N} & 0.9764730 & -0.9281820 & 0.5126760 \\ \mathrm{C} & -0.1560890 & 0.1728590 & 2.1705900 \\ \mathrm{C} & 1.0321880 & -0.4731400 & 1.8077080 \\ \mathrm{H} & 1.9244010 & -0.6288790 & 2.4089340 \\ \mathrm{H} & -0.3842830 & 0.6266510 & 3.1316130 \\ \mathrm{O} & 3.0022500 & -2.2450390 & -0.7407720 \\ \mathrm{H} & 2.8729990 & -3.1845850 & -0.5389410 \\ \mathrm{C} & 2.0784490 & 2.0639530 & -0.5293240 \\ \mathrm{C} & 2.5163870 & 3.1211340 & -0.9252520 \\ \mathrm{H} & 2.9009560 & 4.0563130 & -1.2727750 \\ \mathrm{H} & 1.7005470 & 1.1152050 & -0.1828250\end{array}$

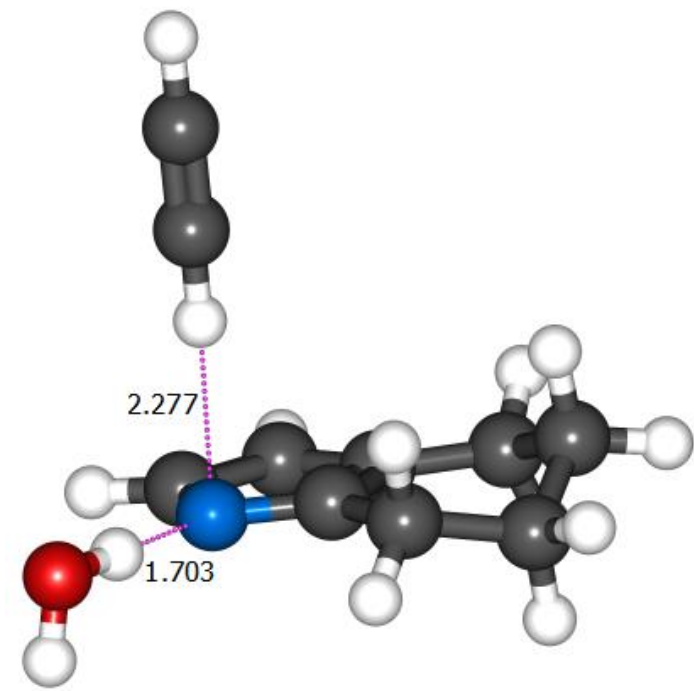

Complex of $1 H$-pyrrole ion, acetylene and water molecule 32

a.u.

$E$ (B2PLYP) $-519.1180563$

$E(\mathrm{~B} 2 \mathrm{PLYP})+Z P E$

$E$ (B2PLYP $)+H_{\text {corr }}$

$E(\mathrm{~B} 2 \mathrm{PLYP})+G_{\text {corr }}$
$-518.9048083$

$-518.8891783$

$-518.9495573$

$\begin{array}{lll}-0.2657370 & -0.0867990 & -0.1140200 \\ -0.7957210 & -1.4673920 & -0.3953540 \\ -2.2863170 & -1.5479170 & -0.0035410 \\ -3.0754020 & -0.3194050 & -0.4902840 \\ -2.5656280 & 0.9824430 & 0.1620310 \\ -1.0583950 & 1.0333790 & 0.1247780 \\ -0.2255820 & -2.2337220 & 0.1495420 \\ -0.6919180 & -1.7196950 & -1.4651770 \\ -2.7288350 & -2.4677040 & -0.4084220 \\ -2.3695010 & -1.6121450 & 1.0918920 \\ -4.1452300 & -0.4510880 & -0.2794780 \\ -2.9748460 & -0.2416750 & -1.5835710 \\ -3.0073110 & 1.8510810 & -0.3486920 \\ -2.9355400 & 1.0287860 & 1.2005760 \\ 1.8968140 & -0.6607580 & 1.3821260 \\ 1.0729680 & 0.2471610 & -0.0605000 \\ -0.1540330 & 2.1243460 & 0.3224340 \\ 1.1284050 & 1.5976130 & 0.1859000 \\ 2.0868390 & 2.1056130 & 0.2360970 \\ -0.4112700 & 3.1584260 & 0.5344340 \\ 2.2780590 & -1.1177880 & 2.1765770 \\ 1.6958930 & -0.8615650 & 2.9087580 \\ 2.4899110 & -0.6033470 & -1.2495440 \\ 3.6044540 & -0.0598980 & -1.5048090 \\ 4.0542220 & 0.8748910 & -1.1966220 \\ 1.8918050 & -1.4764030 & -1.4361320\end{array}$

$-0.0867990$

$-1.4673920$

$-1.5479170$

$-0.3194050$

$-1.7196950$

$-2.4677040$

$-1.6121450$

$-0.4510880$

.2416750

$-0.6607580$

0.2471610

2.1243460

1.5976130

1056130

$-0.0598980$

$-1.4764030$

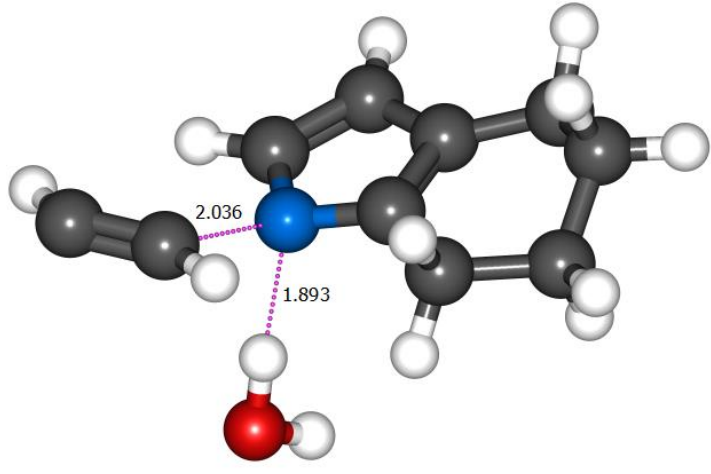

Transition state of 4,5,6,7-tetrahydroindole vinylation $\left(\mathrm{TS}_{32 \rightarrow 33}\right)$ $1356 \mathrm{~cm}^{-1}$

a.u.

$E$ (B2PLYP) $-519.0782124$

$E(\mathrm{~B} 2 \mathrm{PLYP})+Z P E$

$E(\mathrm{~B} 2 \mathrm{PLYP})+H_{c o r r}$ $E(\mathrm{~B} 2 \mathrm{PLYP})+G_{c o r r}$ $-518.8497484$ $-518.9053544$ 


\begin{tabular}{|c|c|c|c|}
\hline $\mathrm{C}$ & 0.6659060 & -0.0925310 & -0.0322060 \\
\hline $\mathrm{C}$ & 0.8745330 & -1.5788190 & -0.0060130 \\
\hline $\mathrm{C}$ & 2.3524520 & -1.8929600 & -0.3265790 \\
\hline C & 3.3212020 & -0.9541900 & 0.4120050 \\
\hline C & 3.1253480 & 0.5132300 & -0.0175560 \\
\hline $\mathrm{C}$ & 1.6603480 & 0.8628740 & -0.0333100 \\
\hline $\mathrm{H}$ & 0.2289090 & -2.0926300 & -0.7307180 \\
\hline $\mathrm{H}$ & 0.6143630 & -1.9902320 & 0.9820020 \\
\hline $\mathrm{H}$ & 2.5635220 & -2.9380020 & -0.0694570 \\
\hline $\mathrm{H}$ & 2.5138620 & -1.7928860 & -1.4095070 \\
\hline $\mathrm{H}$ & 4.3575890 & -1.2626350 & 0.2257420 \\
\hline $\mathrm{H}$ & 3.1524220 & -1.0417830 & 1.4953670 \\
\hline $\mathrm{H}$ & 3.6759450 & 1.1813330 & 0.6585430 \\
\hline $\mathrm{H}$ & 3.5662520 & 0.6630290 & -1.0158810 \\
\hline $\mathrm{H}$ & -3.9275380 & -0.1028040 & 0.0481390 \\
\hline $\mathrm{N}$ & -0.5691580 & 0.5593130 & -0.0332940 \\
\hline $\mathrm{C}$ & 1.0177500 & 2.1442090 & -0.0446340 \\
\hline $\mathrm{C}$ & -0.3415560 & 1.9289470 & -0.0437370 \\
\hline $\mathrm{H}$ & -1.1622950 & 2.6304450 & -0.0512310 \\
\hline $\mathrm{H}$ & 1.5024720 & 3.1133730 & -0.0507040 \\
\hline O & -6.0405860 & -0.9257750 & 0.0561460 \\
\hline $\mathrm{H}$ & -6.9106990 & -1.3603760 & 0.0453910 \\
\hline $\mathrm{C}$ & -1.8185180 & -0.0867220 & -0.0060340 \\
\hline $\mathrm{C}$ & -3.0223800 & 0.5011980 & 0.0349690 \\
\hline $\mathrm{H}$ & -3.1438350 & 1.5810030 & 0.0553320 \\
\hline $\mathrm{H}$ & -1.7326880 & -1.1682450 & -0.0205610 \\
\hline
\end{tabular}

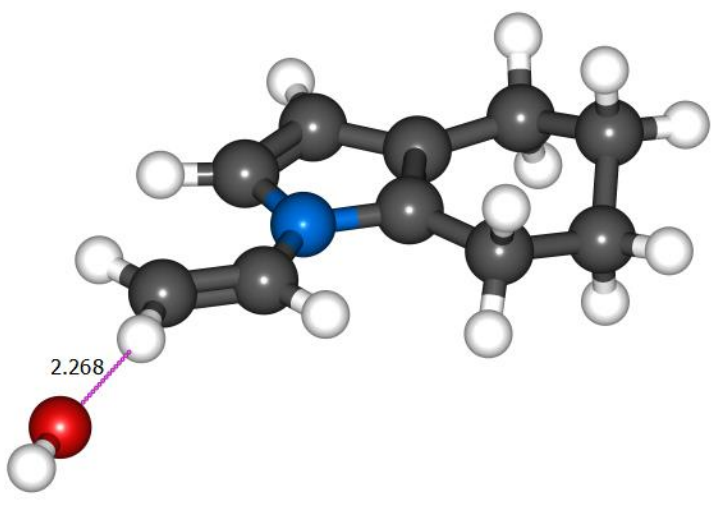
Complex of 1-vinyl-4,5,6,7-tetrahydro- $1 \mathrm{H}$ - indole and hydroxide ion 33

a.u.

$E$ (B2PLYP)

$-519.1043435$

$E(\mathrm{~B} 2 \mathrm{PLYP})+Z P E$

$-518.8853745$

$E(\mathrm{~B} 2 \mathrm{PLYP})+H_{\text {corr }}$

$-518.8713385$

$E(\mathrm{~B} 2 \mathrm{PLYP})+G_{\text {corr }}$

$-518.9257205$
0.0352910
$-0.2006010$
$-1.5637570$
$-0.0180480$
0.6621280
2.1634790
$-1.4427050$
0.0163020
2.8302220
$-0.2559720$
$-0.3260990$
2.2141720
1.0877960
0.3897290
0.7104570
$-0.0475850$
1.0001630
$-0.0449570$
0.1818220
$-2.2496020$
$-0.6943120$
0.5447380
2. 6701220
2.2742070
3. 9087920
2. 7085540
2.5559580
2. 5807730
$-2.0208200$
$-2.3795060$
$-1.3130960$
$-0.2547890$
1.0115110
$-0.0646770$
$-1.4121970$
0.1888370
$-0.3755900$
1.4763170
1.8938960
1.3474600
0.6156150
$-1.0532490$
$-1.3365740$
0.0680450
$-0.2751110$
2. 0423860
1. 4461220
$-1.5141470$
1. 8808810
-2.5024070
-0.0895740
3.1096980
$-2.3414800$
$-1.9479920$
$-0.9086620$
$-1.9193120$
$-0.0078830$
$-0.0607680$
$-0.0371310$
$-0.0367660$
$-0.0832420$
$-3.6648760$
$-0.6952310$
0.0427040
0.0637100
$-4.1132840$
0.2930010
0.0670630
$-4.3365050$
$-1.5457640$
0.0502190
0.1061500

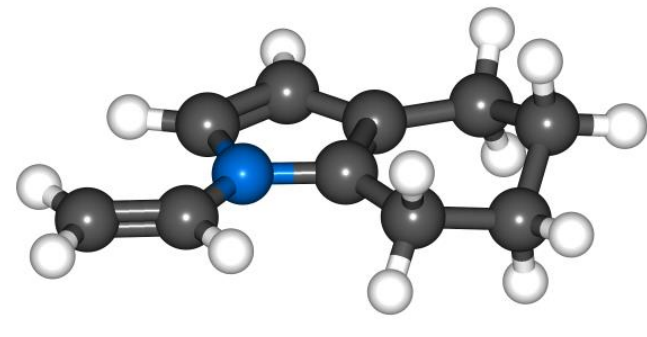

1-vinyl-4,5,6,7-tetrahydro-1H-indole 34

$E$ (B2PLYP)

$E(\mathrm{~B} 2 \mathrm{PLYP})+Z P E$

$E(\mathrm{~B} 2 \mathrm{PLYP})+H_{\text {corr }}$

$E(\mathrm{~B} 2 \mathrm{PLYP})+G_{\text {corr }}$

a.u.

$-443.3194746$

$-443.1106006$

$-443.0999876$

$-443.1455086$ 


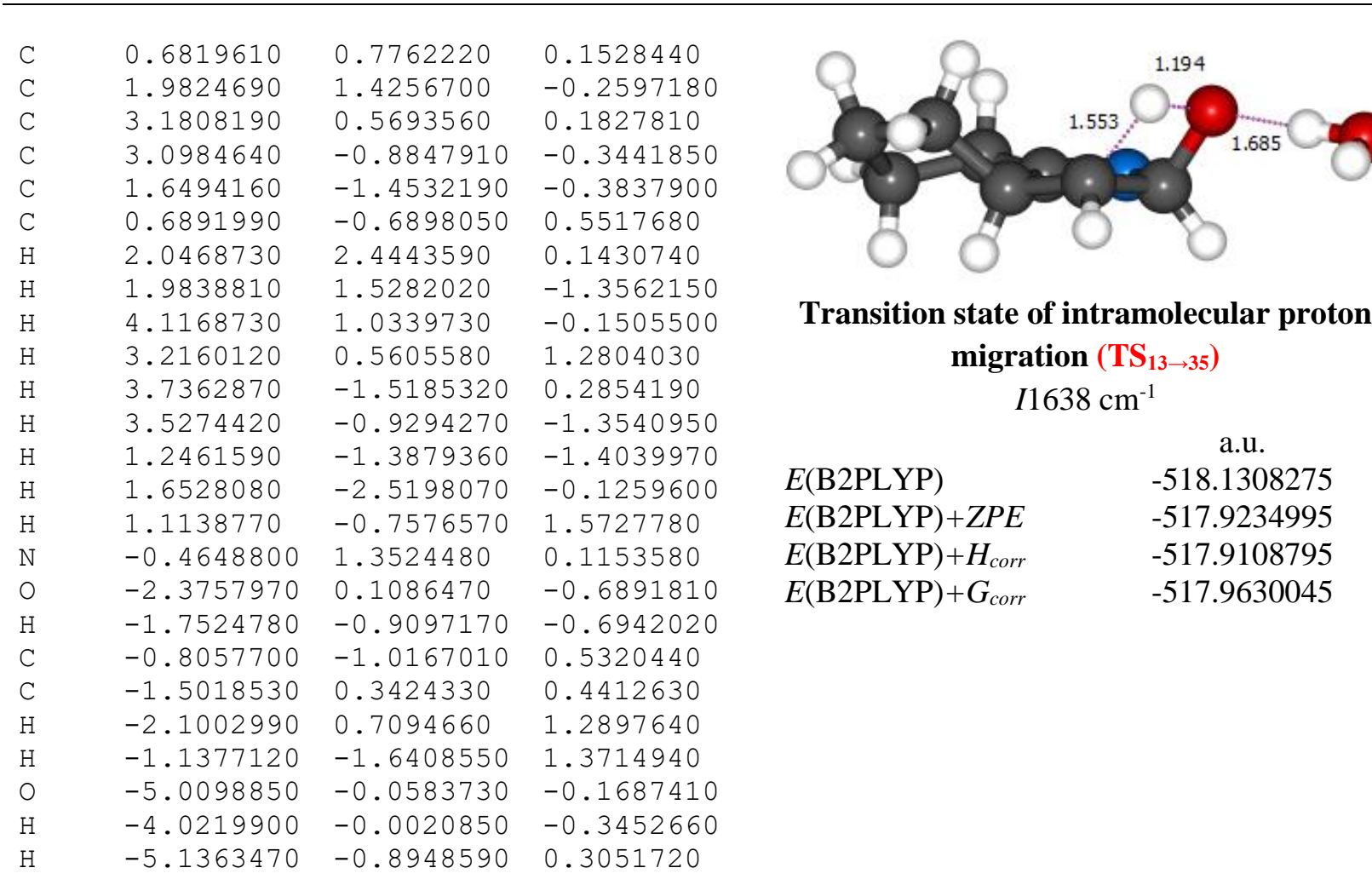

\begin{tabular}{llll}
\hline & & & \\
$\mathrm{C}$ & 0.6791080 & 0.7187050 & 0.1284070 \\
$\mathrm{C}$ & 1.9521750 & 1.4613180 & -0.2004110 \\
$\mathrm{C}$ & 3.1792080 & 0.6824270 & 0.3033580 \\
$\mathrm{C}$ & 3.2274710 & -0.7698030 & -0.2382960 \\
$\mathrm{C}$ & 1.8220950 & -1.4063020 & -0.4380950 \\
$\mathrm{C}$ & 0.7445890 & -0.7571290 & 0.4592350 \\
$\mathrm{H}$ & 1.9234890 & 2.4770400 & 0.2135730 \\
$\mathrm{H}$ & 2.0099500 & 1.5764700 & -1.2943890 \\
$\mathrm{H}$ & 4.0982260 & 1.2152350 & 0.0296740 \\
$\mathrm{H}$ & 3.1509140 & 0.6637640 & 1.4009490 \\
$\mathrm{H}$ & 3.8232690 & -1.3788190 & 0.4541310 \\
$\mathrm{H}$ & 3.7654120 & -0.7849880 & -1.1956310 \\
$\mathrm{H}$ & 1.4984430 & -1.2910520 & -1.4827220 \\
$\mathrm{H}$ & 1.8650550 & -2.4868780 & -0.2507120 \\
$\mathrm{H}$ & 1.1165770 & -0.8467500 & 1.5030870 \\
$\mathrm{~N}$ & -0.4990130 & 1.2249520 & 0.0641880 \\
$\mathrm{O}$ & -2.5154500 & 0.1814690 & -0.7145430 \\
$\mathrm{H}$ & -2.2346620 & -0.5701440 & -1.2805270 \\
$\mathrm{C}$ & -0.7236580 & -1.1664230 & 0.3279060 \\
$\mathrm{C}$ & -1.4719030 & 0.1368470 & 0.3342190 \\
$\mathrm{H}$ & -2.0433710 & 0.4055580 & 1.2402790 \\
$\mathrm{H}$ & -1.0528750 & -1.8435310 & 1.1265950 \\
$\mathrm{O}$ & -5.1608490 & 0.0248460 & 0.0532780 \\
$\mathrm{H}$ & -4.2045280 & 0.0557940 & -0.2083290 \\
$\mathrm{H}$ & -5.2669250 & -0.8147110 & 0.5268840 \\
& & &
\end{tabular}

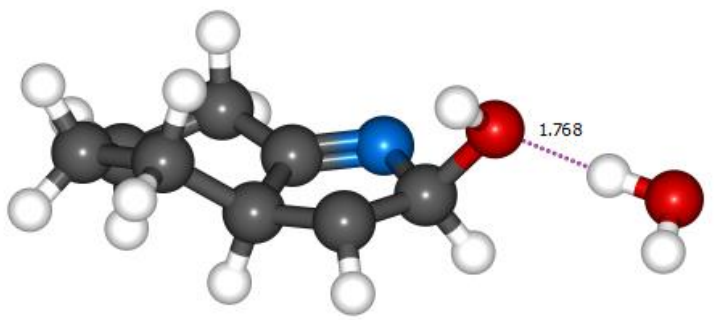

\section{Complex of intermediate carbanion and} water molecule 35

$E$ (B2PLYP)

a.u.

$E(\mathrm{~B} 2 \mathrm{PLYP})+Z P E$

$-518.149016$

$E(\mathrm{~B} 2 \mathrm{PLYP})+H_{\text {corr }}$

$-517.937687$

$E(\mathrm{~B} 2 \mathrm{PLYP})+G_{\text {corr }}$

$-517.977969$ 
$-0.6989430$

$-1.9406250$

$-3.1902160$

$-3.0789850$

$-1.6552880$

$-0.7763270$

$-2.0176150$

$-1.8498920$

$-4.0814260$

$-3.3344140$

$-3.8180720$

$-3.3661690$

$-1.1312310$

$-1.7244290$

$-1.3150920$

0.4890630

2. 4376530

2.1144060

0.6910170

1. 4134370

2. 2265220

1. 0118750

5.0650240

4.0760600

5.1802060
$-0.7460240$

$-1.4694860$

$-0.6128610$

0.8038000

1. 4273920

0.7195990

$-2.4467360$

$-1.6791220$

$-1.1189690$

$-0.5306720$

1. 4519490

0.7601220

1. 3566450

2. 4971550

0.8050470

$-1.2449740$

$-0.1182460$

0.7318990

1.0567260

$-0.1604940$

$-0.4706960$

1.7590390

$-0.0215810$

$-0.0315580$

0.6374190
0.2641560

$-0.1944340$

0.0693310

$-0.5454530$

$-0.4302890$

0.6339950

0.3003290

$-1.2716030$

$-0.3217290$

1.1549730

$-0.0572090$

$-1.6044630$

$-1.3934290$

$-0.1981610$

1. 5975910

0.2634710

$-0.8162480$

$-1.1665170$

0.7924340

0.5978510

1.2581970

1. 5605810

$-0.2807690$

$-0.4736230$

0.4213570

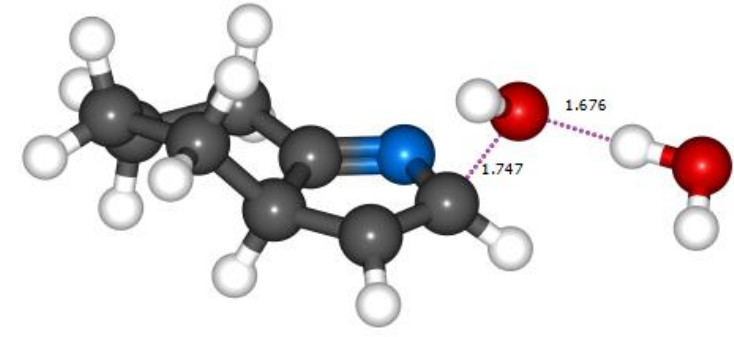

Transition state of hydroxide ion elimination

$$
\left(\mathrm{TS}_{35 \rightarrow 36}\right)
$$

I435 $\mathrm{cm}^{-1}$

E(B2PLYP)

$E(\mathrm{~B} 2 \mathrm{PLYP})+Z P E$

$E(\mathrm{~B} 2 \mathrm{PLYP})+H_{\text {corr }}$

$E(\mathrm{~B} 2 \mathrm{PLYP})+G_{\text {corr }}$ a.u.

$-518.1420519$

$-517.9325399$

$-517.9193859$

$-517.9724089$
1.2730880
2.5578640
3.2576590
2. 2425330
1. 1642930
0.9663590
3. 2162420
2. 3408110
4.0395210
3. 7619350
2.7623590
1. 7505520
0.2096270
1. 4368500
1.6766840
0.3206540
$-3.2917410$
$-2.5595820$
$-0.4060550$
$-0.7096070$
$-1.6095920$
$-1.0117420$
$-5.4482120$
$-4.5398170$
$-5.8755990$
$-0.9722780$
$-0.7574260$
0.5254370
1. 6687510
1.3702560
$-0.1592560$
$-1.6289390$
$-0.6855470$
0.8154690
0.3288700
2. 6012680
1. 8363190
1.8124540
1.8331800
$-0.4761450$
$-1.7908920$
1.3507090
0.7834230
$-0.6711890$
$-1.6109960$
$-2.2113130$
$-0.3511580$
0.0049020
0.5486930
0.1249810
0.2165390
0.9613400
0.4633320
0.2925190
$-0.7843910$
$-1.0181390$
0.8373150
2.0357020
1.1745750
$-0.4931340$
0.0430550
1.2607970
$-0.4806850$
$-1.7390480$
$-1.8006470$
0.5417110
0.4367080
0.1434360
$-1.3525120$
$-0.4329910$
$-0.3554640$
$-2.1920890$
0.5119920
0.4602410
$-0.3498160$

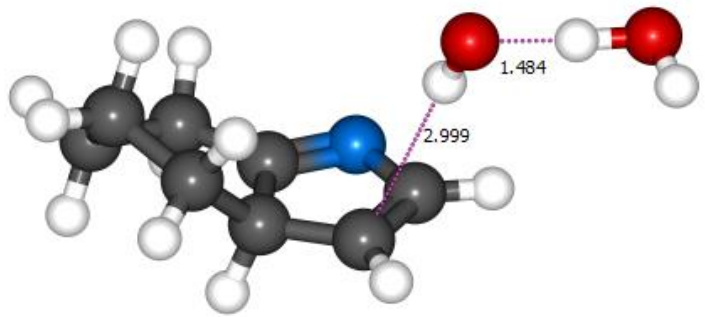

Complex of $\mathbf{3 H}$-pyrrole, hydroxide ion and water molecule 36

a.u.

$E$ (B2PLYP)

$-518.1603674$

$E(\mathrm{~B} 2 \mathrm{PLYP})+Z P E$

$-517.9526354$

$E$ (B2PLYP $)+H_{\text {corr }}$

$-517.9377674$

$E(\mathrm{~B} 2 \mathrm{PLYP})+G_{\text {corr }}$

$-517.9985364$ 
$-0.8603170$

$-2.1331620$

$-3.3522050$

$-3.3454450$

$-1.9274970$

$-0.9217360$

$-2.1474110$

$-2.1455700$

$-4.2752670$

$-3.3609620$

$-3.9983400$

$-3.7909940$

$-1.5521400$

$-1.9724750$

$-1.2571590$

0.3059290

2. 4258650

0.7928500

0.5672100

1. 2731060

1. 5878150

0.8885620

3. 6169570

3.6252340

4.7049260

4.6871740

5.6392400
$-0.7531770$

$-1.4426500$

$-0.6411850$

0.8244310

1. 4658800

0.7091640

$-2.4736730$

$-1.5083810$

$-1.1373360$

$-0.6559770$

1. 4165100

0.8677970

1. 4532600

2. 5196830

0.7604140

$-1.2887590$

$-0.3865570$

1. 5210760

1.0945510

$-0.2749340$

$-0.4948410$

1.8057620

0.0166670

0.1370750

0.2185100

0.1025890

0.4962720
0.0862670

$-0.3238210$

0.1661120

$-0.3346430$

$-0.3903600$

0.4901490

0.0483010

$-1.4225890$

$-0.1543350$

1.2640700

0.3178100

$-1.3362930$

$-1.4224710$

$-0.0915300$

1. 5381940

0.0903680

$-0.3298680$

$-0.5856930$

0.3997020

0.5172360

1. 5471110

1. 1657220

0.2004250

1.2839340

$-0.5509320$

$-1.6309810$

$-0.0756820$

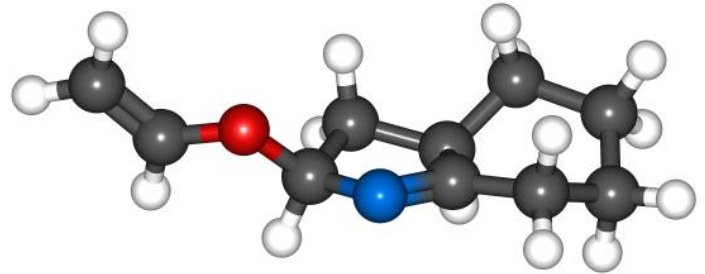

5-vinyloxypyrroline 37

$E$ (B2PLYP)

a.u.

$E(\mathrm{~B} 2 \mathrm{PLYP})+Z P E$

$-519.6961596$

$-519.4600506$

$-519.4477346$

$E$ (B2PLYP $)+H_{\text {corr }}$

$-519.4987706$

$\begin{array}{lll}-0.8291470 & -0.9753260 & -0.0020960 \\ -2.0436560 & -1.1050670 & -0.8574990 \\ -3.1613080 & -0.1896650 & -0.3287330 \\ -2.7202730 & 1.2902350 & -0.2057560 \\ -1.2440420 & 1.4853960 & 0.2550360 \\ -0.6728080 & 0.2116090 & 0.9021010 \\ -2.3582880 & -2.1542420 & -0.9155870 \\ -1.7665040 & -0.8141650 & -1.8840580 \\ -4.0367040 & -0.2600100 & -0.9832160 \\ -3.4790870 & -0.5634110 & 0.6533690 \\ -3.3982030 & 1.7943950 & 0.4923990 \\ -2.8495970 & 1.7877100 & -1.1742680 \\ -0.6201350 & 1.7455720 & -0.6061800 \\ -1.1672890 & 2.3203390 & 0.9595230 \\ -1.2516350 & -0.0048530 & 1.8165030 \\ 0.1957210 & -1.7961290 & 0.0116770 \\ 3.0253470 & -0.6838220 & -0.7875810 \\ 1.4792080 & 0.8703340 & 0.5642290 \\ 0.8118300 & 0.0898440 & 1.2783740 \\ 1.1447420 & -1.2280650 & 0.8389690 \\ 1.9982680 & -1.8150000 & 1.1497660 \\ 1.1363200 & 0.3898220 & 2.2765710 \\ 3.3090320 & 0.4986790 & -0.4378950 \\ 4.2318890 & 0.6664750 & 0.1502700 \\ 2.4281350 & 1.5838500 & -0.5658790 \\ 1.6372230 & 1.5409750 & -1.3121360 \\ 2.7366960 & 2.5705910 & -0.2280000\end{array}$

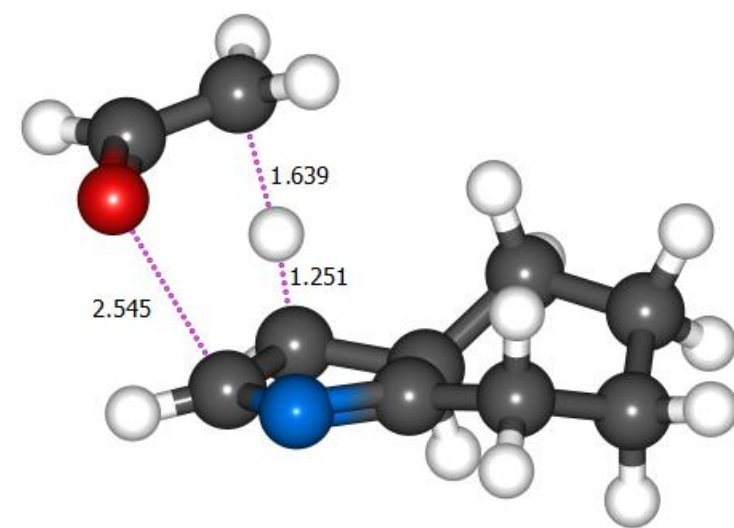

Transition state of acetaldehyde elimination from 5-vinyloxypyrroline $\left(\mathrm{TS}_{37 \rightarrow 38}\right)$ $1613 \mathrm{~cm}^{-1}$

a.u.

$E$ (B2PLYP) $-519.6297704$

$E$ (B2PLYP) $+Z P E$ $-519.4009974$

$E$ (B2PLYP $)+H_{\text {corr }}$

$E(\mathrm{~B} 2 \mathrm{PLYP})+G_{\text {corr }}$

$-519.4386134$ 


\begin{tabular}{|c|c|c|c|c|}
\hline $\mathrm{C}$ & -1.9983300 & -0.7838170 & -0.4604660 & \\
\hline $\mathrm{C}$ & -2.9259210 & -0.0298210 & -1.3678640 & \\
\hline $\mathrm{C}$ & -3.3995120 & 1.2714980 & -0.6866080 & \\
\hline $\mathrm{C}$ & -2.2234550 & 1.9871080 & -0.0004840 & \\
\hline $\mathrm{C}$ & -1.5839640 & 1.1526280 & 1.1434030 & \\
\hline $\mathrm{C}$ & -1.8540780 & -0.3755060 & 0.9861440 & \\
\hline $\mathrm{H}$ & -3.7855320 & -0.6598620 & -1.6369710 & 5.323 \\
\hline $\mathrm{H}$ & -2.4029740 & 0.1953920 & -2.3073190 & \\
\hline $\mathrm{H}$ & -3.8591610 & 1.9291060 & -1.4332740 & \\
\hline $\mathrm{H}$ & -4.1800860 & 1.0437480 & 0.0524740 & \\
\hline $\mathrm{H}$ & -2.5491110 & 2.9580010 & 0.3909440 & \\
\hline $\mathrm{H}$ & -1.4613370 & 2.1994580 & -0.7632290 & Complex of $3 H$-pyrrole and acetaldehyde \\
\hline $\mathrm{H}$ & -0.5025950 & 1.3249500 & 1.1629840 & molecule 38 \\
\hline $\mathrm{H}$ & -1.9716370 & 1.4736390 & 2.1163140 & a.u. \\
\hline $\mathrm{H}$ & -2.8107440 & -0.6022660 & 1.4867700 & -519.6940801 \\
\hline $\mathrm{N}$ & -1.2305740 & -1.7755360 & -0.7923200 & $E(\mathrm{~B} 2 \mathrm{PLYP})+Z P E$ \\
\hline 0 & 5.3413320 & -0.6520840 & -0.9689390 & $E(\mathrm{~B} 2 \mathrm{PLYP})+H_{\text {corr }}$ \\
\hline $\mathrm{H}$ & 4.2048950 & 1.9269300 & 0.3641580 & $E(\mathrm{~B} 2 \mathrm{PLYP})+G_{\text {corr }}$ \\
\hline $\mathrm{C}$ & -0.8060230 & -1.3489500 & 1.4455570 & $E(\mathrm{~B} \angle \mathrm{PLY} \mathrm{P})+\mathrm{G}_{\text {corr }}$ \\
\hline $\mathrm{C}$ & -0.5041940 & -2.1295440 & 0.3873670 & \\
\hline $\mathrm{H}$ & 0.1984910 & -2.9543870 & 0.3373060 & \\
\hline $\mathrm{H}$ & -0.3999860 & -1.4122260 & 2.4482980 & \\
\hline $\mathrm{C}$ & 5.0126720 & -0.0138210 & 0.0185240 & \\
\hline $\mathrm{H}$ & 4.5510140 & -0.5382240 & 0.8790450 & \\
\hline $\mathrm{C}$ & 5.1842140 & 1.4669590 & 0.1755270 & \\
\hline $\mathrm{H}$ & 5.6448370 & 1.9127660 & -0.7096130 & \\
\hline $\mathrm{H}$ & 5.7988330 & 1.6679910 & 1.0632620 & \\
\hline
\end{tabular}

